\title{
Prescribing Topological Defects for the Coupled Einstein and Abelian Higgs Equations
}

\author{
Yisong Yang ${ }^{\star}$ \\ School of Mathematics, Institute for Advanced Study, Princeton, NJ 08540, USA
}

Received: 21 March 1994/in revised form: 28 November 1994

\begin{abstract}
We construct multi-string solutions of the coupled Einstein and Abelian Higgs equations so that the spacetime is uniform along the time axis and a vertical direction and nontrivial geometry is coded on a Riemann surface $M$. We concentrate on the critical Bogomol'nyi phase. When $M$ is compact, the Abelian Higgs model is defined by a complex line bundle $L$ over $M$. We prove that, due to the coupling of the Einstein equations, the Euler characteristic of $M$ and the first Chern number of the line bundle $L$ identified as the total string number impose an exact obstruction to the existence of a string solution. Such an obstruction leads to some interesting implications. We then study the existence of multi-string solutions which can realize a prescribed string distribution. We show that there are such solutions when the local string winding numbers do not exceed half of the total string number. When $M$ is noncompact and globally conformal to a plane, we show that the energy scale of symmetry breaking plays a crucial role and there are finite-energy radially symmetric string solutions realizing a given string number if and only if the symmetry breaking scale is sufficiently small but nonvanishing. Finally, we obtain finite-energy multistring solutions with an arbitrary string distribution and associated local winding numbers. These solutions are not radially symmetric and are regular everywhere and topologically nontrivial so that both the energy of the matter-gauge sector and the energy of the gravitational sector viewed as the total Gauss curvature of $M$ are quantized.
\end{abstract}

\section{Introduction}

Domain walls, strings, and monopoles are interesting topological defects arising as static solutions of gauge field equations with broken symmetry and nontrivial topology. When the Einstein equations are coupled into the theory, these solutions give rise to various cosmological implications. Cosmic strings are static solutions of the coupled Einstein and Yang-Mills-Higgs equations so that the spacetime is uniform

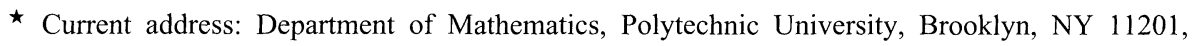
USA 
along the time axis as well as a vertical direction. When the gauge group is Abelian, the system represents the coupling of gravity and a condensed matter system (superconductivity). In this framework, cosmic strings are static vortex-like solutions which are believed to be relevant in the theory of galaxy formation in the early universe $[\mathrm{K} 1, \mathrm{~V}, \mathrm{G}, \mathrm{W}, \mathrm{Gr}]$. A basic perception is that cosmic strings might generate the necessary density perturbations and curvature concentrations from which galaxies evolve. For a comprehensive but nontechnical survey article, see [K2]. See also [Bra].

The main purpose of this paper is to construct cosmic strings solutions of the coupled Einstein and Abelian Higgs equations in the Bogomol'nyi phase. These solutions realize an arbitrarily prescribed string distribution.

It may be instructive to recall that, in the absence of gravity, the Abelian Higgs action density in suitably normalized units is given by the expression [NO, JT]

$$
\mathscr{L}=\frac{1}{4} F_{\mu \nu} F^{\mu \nu}+\frac{1}{2}\left(D_{\mu} \phi\right)\left(D^{\mu} \phi\right)^{*}+\frac{\lambda}{8}\left(|\phi|^{2}-\varepsilon^{2}\right)^{2},
$$

where $\phi$ is a complex scalar, $A_{\mu}$ a real vector potential, $F_{\mu v}=\partial_{\mu} A_{v}-\partial_{v} A_{\mu}$ the Maxwell field tensor, $D_{\mu}=\partial_{\mu}-\mathrm{i} A_{\mu}$ the gauge-covariant derivative, the flat Minkowski metric $\operatorname{diag}(-1,1,1,1)$ is used to raise or lower indices, and the constant $\varepsilon>0$ measures the energy scale of symmetry breaking of the model. The dimensionless parameter $\lambda>0$ is known to characterize the types of superconductivity. The case $\lambda<1$ corresponds to type-I superconductivity, while $\lambda>1$, type-II, characterized by the existence of a sublevel critical magnetic field. The situation $\lambda=1$ is the intermediate type called the Bogomol'nyi critical phase which is characterized by a zero surface-energy and only in such a situation can all string-like solutions be constructed [JT, T1, T2] due to a reduction from the second-order equations to a first-order system [B]. In this case the vortex or string number $N$ is a topological invariant characterizing the homotopy class of the solutions in the fundamental group $\pi_{1}\left(S^{1}\right)=\mathbf{Z}$ and there are no restrictions to the ranges of the string number $N$ and the symmetry breaking scale $\varepsilon$.

In the present paper, it will be seen that some new phenomena take place when gravity is put into the model through the coupling of the Einstein equations. Since we are interested in cosmic strings solutions, the Minkowski spacetime takes the form $\mathbf{R}^{1,1} \times M$, where $M$ may be assumed to be a Riemann surface equipped with an unknown gravitational metric which will be determined by the coupled Einstein and Abelian Higgs equations. We will concentrate on the critical Bogomol'nyi phase $\lambda=1$. The case $\lambda \neq 1$ is more difficult and will be pursued elsewhere.

The following two types of results will be presented.

I. Exact obstructions to the ranges of $N$ and $\varepsilon$ for the existence of an $N$-string solution. When $M$ is compact, the obstruction is related to topology. When $M$ is noncompact, the obstruction comes from the finite-energy requirement or geodesic completeness.

II. Existence theorems for multi-string solutions. We aim at getting static regular solutions that can realize a prescribed string distribution. These solutions are nonradially symmetric, thus, may not be obtained by a dynamical system approach.

In Sect. 1, we consider the case that $M$ is compact. In this circumstance the Abelian Higgs sector is defined by a complex line bundle $L$ over $M$ and the string number is the first Chern number of $L$. The bundle $L$ reflects the magnetic excitation pattern of the gauge and matter fields. We show that the Euler characteristic 
$\chi(M)$ of $M$ and the first Chern number $c_{1}(L)$ of $L$ or the string number $N$ impose an explicit constraint on the symmetry-breaking parameter $\varepsilon$ for the existence of a solution. Under our notation there, the constraint reads $\chi(M)=2 \pi \varepsilon^{2} G N$, where $G$ is Newton's gravitational constant. This equation relates the topology of the underlying gravitational surface $M$ to the topology $\left(N=\left|c_{1}(L)\right|\right)$ of the magnetic excitation pattern of the matter-gauge sector. In particular, we observe that there are only countably many values of $\varepsilon$ accumulated at $\varepsilon=0$ to permit the existence of cosmic strings. We then state some existence results for multi-string solutions. For the prescribed string problem, our sufficient condition for existence requires that the local winding numbers do not exceed half of the total string number $N$.

In Sect. 2, we consider the case that $M$ is noncompact. We shall assume that $M$ is conformally $\mathbf{R}^{2}$. We have already found in [CHMcY] that, for self-dual equations $[\mathrm{CG}, \mathrm{L}]$, the energy scale of symmetry breaking imposes an obstruction to the string number $N$. We shall prove the equivalence of the self-dual equations and the second-order Einstein and Abelian Higgs equations under the cylindrical symmetry assumption, regardless of the asymptotic behavior of the metric. Therefore we arrive at a true obstruction to the topological charge $N$ for finite-energy cosmic strings. Roughly speaking, the obstruction is less stringent for small scales of symmetry breaking. This observation raises the question whether there are string solutions when symmetry is restored. We shall show that then, there will be no finite-energy string solutions. There, again, we state an existence theorem for multi-string solutions. The basic assumption reads $N \leqq 1 / 2 \pi \varepsilon^{2} G$ which imposes no restrictions to the local winding number. The borderline $N=1 / 2 \pi \varepsilon^{2} G$ is a special situation that the choice of a background metric becomes crucial, which signals the appearance of some obstructions when $N$ is large or gravitation is strong (large $G$ ). In particular, beyond this barrier, metric incompleteness will occur.

Section 3 studies what happens when strings are absent. We will show in the compact case and for the general coupling parameter $\lambda>0$ that in this situation there will be only trivial solutions and $M$ must be a flat torus. In other words, the absence of string defects implies the absence of gravity.

Section 4 is a proof of the existence theorem for multi-string solutions on a compact Riemann surface $M$. The Einstein equations reduce to a scalar equation relating the unknown Gauss curvature of $M$ to the energy density of the Abelian Higgs sector while the string or vortex equations are sitting in the unknown gravitational background. In such a setting, prescribing $N$ strings is like prescribing a Gauss curvature through the influence of matter-gauge fields, while the former also determines the latter through the background metric. We use a standard device to reduce the unknown metric to an unknown conformal factor. Under the topological constraint $\chi(M)=2 \pi \varepsilon^{2} G N$, we can combine the Einstein and Abelian Higgs equations into a single elliptic scalar equation with an adjustable free parameter which may be used to get a subsolution as was done earlier in a different problem [CY]. The difficulty in getting a classical supersolution forces us to introduce a $\delta$-perturbed version of the equation. We then pass to the $\delta \rightarrow 0$ limit of the solution sequence of the $\delta$-equations. There we see that, in order to acquire $L^{p}$-convergence for some $p>1$, we need to assume that the local winding numbers are all below $N / 2$. In particular it follows that, when $N \geqq 3$, there are $N$-string solutions over $M$ if and only if the condition $\chi(M)=2 \pi \varepsilon^{2} \bar{G} N$ holds.

In Sect. 5, we prove the existence of multi-string solutions on a conformally flat surface. Again the method is to construct sub/supersolution pairs for a family of $\delta$-regularized equations. It is interesting to observe that the condition $N \leqq 1 / 2 \pi \varepsilon^{2} G$ 
plays crucial roles both locally at the string axises in order to achieve an $L^{p}$ convergence $(p>1)$ and asymptotically at infinity in order to make an adjustable term dominant to allow the existence of a special subsolution. Note that, here, we do not have a suitable variational principle to work with.

In Sect. 6, we complete our proof of existence by showing that the solutions obtained in Sect. 6 are of finite energies. More precisely, we establish sharp decay estimates for the physical quantities at infinity when $N<1 / 2 \pi \varepsilon^{2} G$. The solutions are in the category that the Higgs field approaches the asymmetric vacuum $|\phi|=1$ at infinity. Nevertheless, the gravitational metric vanishes asymptotically like $r^{-4 \pi \varepsilon^{2} G N}$ which results in the property that the Gauss curvature, the magnetic field, the kinetic energy of $\phi$, and the Higgs potential all go to zero at infinity faster than any power function of the type $r^{-b}(b>0)$. Hence both the total curvature and the Abelian Higgs energy are quantized. At the borderline $N=1 / 2 \pi \varepsilon^{2} G$, our estimates still ensure curvature and energy quantizations and metric completeness.

In a broader sense, this work is related to the problem of constructing regular static solutions of the Einstein equations coupled with matter and gauge fields. It is well known that the Schwarzschild blackhole is the only solution of the vacuum Einstein equations which is singular somewhere. When coupled with the Maxwell equations, the only solution is the Reissner-Nordström solution which is again singular somewhere. Recently, it is shown in [BMc, SWYMc] that, when the Einstein equations are coupled with the $S U(2)$ Yang-Mills fields, there exist static regular solutions. All the above-mentioned solutions are radially symmetric. Our solutions constructed here are all static finite-energy regular solutions. Besides, they are nonradially symmetric and carry nontrivial topology.

It has been observed in [BMc] that the existence of regular static solutions of the coupled Einstein and Yang-Mills equations in three space dimensions indicates that the weak gravitational effect cannot be neglected. The obstruction theorems in the present paper provide further evidence to the above observation. For example, the inequality $N \leqq 1 / 2 \pi \varepsilon^{2} G$ in the noncompact case imposes a very weak upper threshold to admissible string numbers $N$ since $G$ is a small quantity. However, because $G \neq 0$, such a constraint must not be ignored in order to avoid energy blow-ups. There is no such a phenomenon when gravity is absent [JT].

\section{The Case of a Compact Surface}

We divide our discussion into three subsections. We first introduce quickly the coupled Einstein and Abelian Higgs equations in the aforementioned two-dimensional setting of current interest. We then establish an obstruction theorem and point out its important consequences. Finally we state our existence theorem for the prescribed string solutions.

1.1. The Governing Equations. We begin by assuming that $g_{\mu v}$ is a general metric tensor with signature $(-+++), R_{\mu v}$ the Ricci tensor, and $R$ the scalar curvature. Recall that the Einstein tensor takes the form

$$
G_{\mu \nu}=R_{\mu \nu}-\frac{1}{2} g_{\mu \nu} R
$$

In order to define the Abelian Higgs sector properly, we need to work in a frame-work in which the Higgs field $\phi$ is a cross-section on a $U(1)$-line bundle 
$L$ over the spacetime and the gauge field, say $A$, is a connection 1-form. Suppose that $h$ is a Hermitian metric of $L$ and $\left\{\left(U_{\alpha}, e_{\alpha}\right)\right\}$ is such an atlas of local trivializations of $L$ that $e_{\alpha}$ satisfies $h\left(e_{\alpha}, e_{\alpha}\right)=1, \forall \alpha$. Let $\phi_{\alpha}$ be the local representation of $\phi$ on $U_{\alpha}: \phi=\phi_{\alpha} e_{\alpha}$. Then we have $h(\phi, \phi)=\left|\phi_{\alpha}\right|^{2}$ which is obviously a localchart-independent scalar field, thus, may conveniently be denoted by $|\phi|^{2}$. Therefore, using local coordinates and local representations, we can write the Abelian Higgs action density at the Bogomol'nyi phase $\lambda=1$ in the form

$$
\mathscr{L}=\frac{1}{4} g^{\mu \mu^{\prime}} g^{\nu v^{\prime}} F_{\mu \nu} F_{\mu^{\prime} v^{\prime}}+\frac{1}{2} g^{\mu \nu}\left(D_{\mu} \phi\right)\left(D_{\nu} \phi\right)^{*}+\frac{1}{8}\left(|\phi|^{2}-\varepsilon^{2}\right)^{2},
$$

where and in the sequel we also allow the vanishing of the symmetry-breaking parameter, $\varepsilon=0$, to include in the model the restoration of symmetry. Note that, in the above expression and subsequent discussion, we suppress the subscript " $\alpha$ " when there is no risk of confusion, and that, $D$ is the covariant derivative induced from the gauge connection $A$ and $F=\mathrm{d} A$ is the curvature of $A$ or the Maxwell field. The presence of the gravitational metric $g_{\mu v}$ indicates the influence of gravity.

The Einstein equations coupled with the Abelian Higgs model are ${ }^{1}$

$$
\begin{aligned}
G_{\mu v} & =-4 \pi G T_{\mu v}, \\
\frac{1}{\sqrt{|g|}} D_{\mu}\left(g^{\mu v} \sqrt{|g|}\left[D_{v} \phi\right]\right) & =\frac{1}{2}\left(|\phi|^{2}-\varepsilon^{2}\right) \phi, \\
\frac{1}{\sqrt{|g|}} \partial_{\mu^{\prime}}\left(g^{\mu v} g^{\mu^{\prime} v^{\prime}} \sqrt{|g|} F_{v v^{\prime}}\right) & =\frac{\mathrm{i}}{2} g^{\mu v}\left(\phi\left[D_{v} \phi\right]^{*}-\phi^{*}\left[D_{v} \phi\right]\right),
\end{aligned}
$$

where $G$ is Newton's gravitational constant (or more precisely a dimensionless rescaling factor of the gravitational constant) and

$$
T_{\mu v}=g^{\mu^{\prime} v^{\prime}} F_{\mu \mu^{\prime}} F_{v v^{\prime}}+\frac{1}{2}\left(\left[D_{\mu} \phi\right]\left[D_{v} \phi\right]^{*}+\left[D_{\mu} \phi\right]^{*}\left[D_{v} \phi\right]\right)-g_{\mu v} \mathscr{L}
$$

is the energy-momentum tensor of the Abelian Higgs sector obtained by varying the gravitational metric.

We assume that the spacetime is uniform along the time axis $x^{0}$ and the $x^{3}$ direction so that the line element takes the form

$$
\begin{aligned}
\mathrm{d} s^{2} & =g_{\mu \nu} \mathrm{d} x^{\mu} \mathrm{d} x^{\nu} \\
& =-\mathrm{d} t^{2}+\mathrm{d} z^{2}+g_{j k} \mathrm{~d} x^{j} \mathrm{~d} x^{k}, \quad j, k=1,2,
\end{aligned}
$$

where $t=x^{0}, z=x^{3}$, and $g_{j k}$ is the Riemannian metric tensor of an orientable 2-surface $M$ (without boundary), and that $A_{\mu}, \phi$ depend only on the coordinates on $M$ and

$$
A_{\mu}=\left(0, A_{1}, A_{2}, 0\right)
$$

1 In literature, the matter-coupling factor $4 \pi G$ in the Einstein equations is often written $8 \pi G$. Here we still use $4 \pi G$ in order to be consistent with our notation in [CHMcY]. 
Then $T_{\mu \nu}$ is simplified to

$$
\begin{aligned}
& T_{00}=\mathscr{E}, \quad T_{33}=-\mathscr{E}, \quad T_{03}=T_{0 j}=T_{3 j}=0, \\
& T_{j k}=g^{j^{\prime} k^{\prime}} F_{j j^{\prime}} F_{k k^{\prime}}+\frac{1}{2}\left(\left[D_{j} \phi\right]\left[D_{k} \phi\right]^{*}+\left[D_{j} \phi\right]^{*}\left[D_{k} \phi\right]\right)-g_{j k} \mathscr{E},
\end{aligned}
$$

where

$$
\mathscr{E}=\frac{1}{4} g^{j j^{\prime}} g^{k k^{\prime}} F_{j k} F_{j^{\prime} k^{\prime}}+\frac{1}{2} g^{j k}\left(D_{j} \phi\right)\left(D_{k} \phi\right)^{*}+\frac{1}{8}\left(|\phi|^{2}-\varepsilon^{2}\right)^{2}
$$

is the energy density of the Abelian Higgs sector which is now defined by the line bundle $L$ restricted to the 2-surface $M$. The Maxwell field $F_{j k}$ represents the first Chern class, of course. Besides, if we use $K_{g}$ to denote the Gauss curvature of $\left(M,\left\{g_{j k}\right\}\right)$, the Einstein tensor reduces under local isothermal coordinates into the form

$$
\begin{aligned}
-G_{00} & =G_{33}=K_{g}, \\
G_{\mu v} & =0 \text { for other values of } \mu, v .
\end{aligned}
$$

As a consequence, the system becomes the following two-dimensional Einstein and Abelian Higgs equations on $M$ :

$$
\begin{aligned}
K_{g} & =4 \pi G \mathscr{E}, \quad T_{j k}=0, \\
\frac{1}{\sqrt{g}} D_{J}\left(g^{j k} \sqrt{g}\left[D_{k} \phi\right]\right) & =\frac{1}{2}\left(|\phi|^{2}-\varepsilon^{2}\right) \phi, \\
\frac{1}{\sqrt{g}} \partial_{j^{\prime}}\left(g^{j k} g^{j^{\prime} k^{\prime}} \sqrt{g} F_{k k^{\prime}}\right) & =\frac{\mathrm{i}}{2} g^{j k}\left(\phi\left[D_{k} \phi\right]^{*}-\phi^{*}\left[D_{k} \phi\right]\right),
\end{aligned}
$$

where $g$ also stands for the determinant formed from $\left\{g_{j k}\right\}$.

1.2. The Obstruction Theorem. The system (1) describes the interaction of the gravitational and gauge-matter sectors confined in a two-dimensional space. We will see that these two sectors are so strongly coupled that, topologically, they totally determine one another. In fact, the first Chern number

$$
c_{1}(L)=\frac{1}{4 \pi} \int_{M} \varepsilon^{j k} F_{j k} \mathrm{~d} \Omega_{g}
$$

classifies the line bundle $L$ up to isomorphisms which clearly indicates the magnetic excitation pattern of the theory because the integer $N=\left|c_{1}(L)\right|$ is the number of magnetic strings through $M$, identified as the algebraic number of zeros of the order parameter $\phi$. On the other hand, the Gauss curvature $K_{g}$ reflects the topology of $M$ and measures the gravitational strength, which, by the first equation in (1), is determined by the energy distribution of the matter and gauge fields. Thus it is clear that there must be a link between these structures through the coupling of gravity with the matter-gauge fields such as that given in (1).

More precisely, calling a solution of (1) with $\left|c_{1}(L)\right|=N$ an $N$-string, we have the following basic result. 
Theorem 1.1. Given an integer $N \geqq 0$, there exists an $N$-string solution for the Einstein and Abelian Higgs equations (1) on a line bundle L equipped with a certain Hermitian structure over an appropriate compact Riemann surface $M$, only if the string number $N$, the first Chern number $c_{1}(L)$ of $L$, the Euler characteristic $\chi(M)$ of $M$, the symmetry-breaking parameter $\varepsilon>0$, and the gravitational coupling factor $G$ satisfy the exact relation

$$
\chi(M)=2 \pi \varepsilon^{2} G N=2 \pi \varepsilon^{2} G\left|c_{1}(L)\right| .
$$

Furthermore, if $N \geqq 3$, the condition (2) is necessary and sufficient for the existence of an $N$-string solution. In any case, the solutions of (1) can all be obtained from a self-dual or anti-self-dual system in which the matter-gauge equations are all of the first order.

Before going into the proof of the above theorem, we would like to point out some of its interesting implications.

1. The Unique Topology of the Underlying Surface. It is well known that a compact orientable 2-surface $M$ is topologically a sphere with $n$ handles and the number $n$ is called the genus of $M$. The Euler characteristic satisfies the equation $\chi(M)=2-2 n$ (see e.g. [NS]). Thus the relation (2) implies that the only possible situation we may have so that the Einstein and Abelian Higgs system (1) has a cosmic string solution is given by $n=0$. In other words, the 2 -surface must be diffeomorphic to the Riemann sphere $S^{2}$ and all other geometries with $n \neq 0$ are ruled out. In particular, the surface $M$ cannot be a torus $(n=1)$. This result implies the nonexistence of gravitational string condensation realized by the appearance of a periodic lattice structure.

2. The Quantization of Symmetry Breaking Scale. Now we use the conclusion $\chi(M)=2$ (or $n=0$ ) arrived above and rewrite (2) in the form

$$
\varepsilon=\varepsilon_{N}=\frac{1}{\sqrt{\pi G N}}, \quad N=1,2, \ldots .
$$

We view the gravitational constant $G$ as fixed. Equation (3) says that there are only countably many levels of the symmetry breaking scale $\varepsilon$ for which there may exist cosmic string solutions, and, when $\varepsilon$ is away from those quantized levels, there will be no strings. In particular, when $\varepsilon>\varepsilon_{1}=1 / \sqrt{\pi G}$, there is nonexistence. Such a fact seems to suggest that the existence of string solutions prefers lower values of the symmetry breaking scale $\varepsilon$. Indeed, in (2), the vanishing of $\varepsilon$ implies that $M$ is topologically a torus and (2) no longer presents a constraint to the string number $N$. However, we will see that in this case there is no nontrivial solutions. This simple fact will be established later. Thus, we observe that the existence of cosmic strings indeed requires symmetry breaking. Such a fact is also true for the noncompact case.

3. Effective Radius vs. Gravitational Attraction. Finally, since $M$ is topologically a sphere, we may define the "effective radius" of $M$, say $R_{\mathrm{eff}}$, by setting $4 \pi R_{\text {eff }}^{2}=|M|_{g}$. Then we find that $R_{\text {eff }}$ has the lower bound

$$
R_{\mathrm{eff}}>\frac{1}{\varepsilon^{2} \sqrt{\pi G}}
$$


This simple inequality says that, as the gravitational constant, a smaller value of the symmetry-breaking scale leads to a larger $R_{\text {eff }}$. Thus it looks as if $\varepsilon$ make an effective contribution to the attractive gravitational force.

Proof of Theorem 1.1. Following the work of [CG, L], it is known that Eqs. (1) possess the following Bogomol'nyi self-dual structure

$$
\begin{aligned}
K_{g}-4 \pi G \mathscr{E} & =0, \\
D_{j} \phi \pm \mathrm{i} \varepsilon_{j}^{k} D_{k} \phi & =0, \\
\varepsilon^{j k} F_{j k} \pm\left(|\phi|^{2}-\varepsilon^{2}\right) & =0 .
\end{aligned}
$$

Namely, the solutions of (5) verify (1). Here $\varepsilon_{j k}$ is the standard Levi-Civita skewsymmetric 2 -tensor satisfying $\varepsilon_{12}=\sqrt{g}$.

We first show that the systems (1) and (5) are actually equivalent. Note that a formally special case of the equivalence has been studied in [Y1]. This equivalence problem is of interest in a broader sense in general gauge theories. For Yang-Mills with non-Abelian gauge groups, the equivalence is false $[\mathrm{Bu}, \mathrm{T} 3, \mathrm{SSU}, \mathrm{P}, \mathrm{BM}, \mathrm{SS}, \mathrm{Bo}]$. In our problem here, the equivalence of (1) and (5) is the crucial point.

Use $\Delta_{g}$ to denote the Laplace-Beltrami operator with respect to the unknown metric $g=\left\{g_{j k}\right\}$ with the sign convention that, on a compact surface, all its nonzero eigenvalues are negative. Rewrite the last equation in (1) as

$$
\partial_{j}\left(\varepsilon^{J^{\prime} k^{\prime}} F_{J^{\prime} k^{\prime}}\right)+\mathrm{i} \varepsilon_{j j^{\prime}} g^{j^{\prime} k^{\prime}}\left(\phi\left[D_{k^{\prime}} \phi\right]^{*}-\phi^{*}\left[D_{k^{\prime}} \phi\right]\right)=0 .
$$

Therefore

$$
\frac{1}{\sqrt{g}} \partial_{j}\left(\sqrt{g} g^{j k} \partial_{k}\left[\varepsilon^{J^{\prime} k^{\prime}} F_{j^{\prime} k^{\prime}}\right]\right)+\frac{\mathrm{i}}{\sqrt{g}} \partial_{J}\left(\sqrt{g} \varepsilon^{j k}\left[\phi\left(D_{k} \phi\right)^{*}-\phi^{*}\left(D_{k} \phi\right)\right]\right)=0
$$

Thus

$$
\Delta_{g}\left(\varepsilon^{j k} F_{j k}\right)-|\phi|^{2}\left(\varepsilon^{j k} F_{j k}\right)+2 \mathrm{i} \varepsilon^{j k}\left(D_{j} \phi\right)\left(D_{k} \phi\right)^{*}=0 .
$$

Besides, we also have by using the second equation in (1) that

$$
\Delta_{g}|\phi|^{2}=|\phi|^{2}\left(|\phi|^{2}-\varepsilon^{2}\right)+2 g^{j k}\left(D_{j} \phi\right)\left(D_{k} \phi\right)^{*} \text {. }
$$

These equations give us the useful expression

$$
\begin{aligned}
\Delta_{g}\left(\varepsilon^{j k} F_{j k} \pm\left[|\phi|^{2}-\varepsilon^{2}\right]\right)= & |\phi|^{2}\left(\varepsilon^{j k} F_{j k} \pm\left[|\phi|^{2}-\varepsilon^{2}\right]\right) \\
& \pm g^{j k}\left(D_{j} \phi \pm \mathrm{i} \varepsilon_{j}^{j^{\prime}} D_{j^{\prime}} \phi\right)\left(D_{k} \phi \pm \mathrm{i} \varepsilon_{k}^{k^{\prime}} D_{k^{\prime}} \phi\right)^{*} .
\end{aligned}
$$

Define for a solution triplet of (1) the quantities

$$
P^{+}=\varepsilon^{j k} F_{j k}+\left(|\phi|^{2}-\varepsilon^{2}\right) \text { and } P^{-}=\varepsilon^{j k} F_{j k}-\left(|\phi|^{2}-\varepsilon^{2}\right) \text {. }
$$

Thus, according to the first equation in (1), we have, after a lengthy calculation,

$$
P^{+} P^{-}=4 g^{j k} T_{j k} \equiv 0 \text {. }
$$


The first part of (6) implies the elliptic inequality

$$
\begin{aligned}
\Delta_{g} P^{+} & =|\phi|^{2} P^{+}+g^{j k}\left(D_{j} \phi+\mathrm{i} \varepsilon_{j}^{j^{\prime}} D_{\jmath^{\prime}} \phi\right)\left(D_{k} \phi+\mathrm{i} \varepsilon_{k}^{k^{\prime}} D_{k^{\prime}} \phi\right)^{*} \\
& \geqq|\phi|^{2} P^{+} .
\end{aligned}
$$

Since $M$ is compact, the maximum principle implies that, either $P^{+} \equiv 0$ or $P^{+}<0$ on $M$.

Similarly, the second part of (6) implies

$$
\begin{aligned}
\Delta_{g} P^{-} & =|\phi|^{2} P^{-}-g^{j k}\left(D_{j} \phi-\mathrm{i} \varepsilon_{j}^{j^{\prime}} D_{J^{\prime}} \phi\right)\left(D_{k} \phi-\mathrm{i} \varepsilon_{k}^{k^{\prime}} D_{k^{\prime}} \phi\right)^{*} \\
& \leqq|\phi|^{2} P^{-} .
\end{aligned}
$$

The maximum principle again says that, either $P^{-} \equiv 0$ or $P^{-}>0$.

Using these observations in (7) we see that either $P^{+} \equiv 0$ or $P^{-} \equiv 0$. Accordingly, we have either $D_{j} \phi+\mathrm{i} \varepsilon_{j}^{k} D_{k} \phi \equiv 0$ or $D_{j} \phi-\mathrm{i} \varepsilon_{j}^{k} D_{k} \phi \equiv 0$. Thus (5) is fulfilled and the two systems (1) and (5) are equivalent.

As a consequence, we may concentrate on the system (5).

It is easily seen that the energy density $\mathscr{E}$ of the Abelian Higgs theory can be rewritten in the form

$$
\begin{aligned}
\mathscr{E}= & \frac{1}{4} g^{\prime j^{\prime}} g^{k k^{\prime}}\left(F_{j k} \pm \frac{1}{2} \varepsilon_{j k}\left(|\phi|^{2}-\varepsilon^{2}\right)\right)\left(F_{j^{\prime} k^{\prime}} \pm \frac{1}{2} \varepsilon_{j^{\prime} k^{\prime}}\left(|\phi|^{2}-\varepsilon^{2}\right)\right) \\
& +\frac{1}{4} g^{j k}\left(D_{j} \phi \pm \mathrm{i} \varepsilon_{j}^{J^{\prime}} D_{j^{\prime}} \phi\right)\left(D_{k} \phi \pm \mathrm{i} \varepsilon_{k}^{k^{\prime}} D_{k^{\prime}} \phi\right)^{*} \\
& \pm \frac{1}{4} \varepsilon^{2} \varepsilon^{j k} F_{j k} \pm \nabla_{j}\left(\varepsilon^{j k} J_{k}\right),
\end{aligned}
$$

where $\nabla_{J}$ is the covariant derivative with respect to the metric $\left\{g_{J k}\right\}$ and $J_{k}$ is the current vector defined by

$$
J_{k}=\frac{\mathrm{i}}{4}\left(\phi\left[D_{k} \phi\right]^{*}-\phi^{*}\left[D_{k} \phi\right]\right) .
$$

Hence the curvature equation in (5) becomes

$$
K_{g}= \pm \pi \varepsilon^{2} G \varepsilon^{j k} F_{j k} \pm 4 \pi G \nabla_{j}\left(\varepsilon^{j k} J_{k}\right), \quad x \in M .
$$

We are now ready to determine the relation between the topology of $M$ and the string number of a solution.

For given $p \in M$, choose a specific isothermal coordinate system $\left(U,\left(x^{\jmath}\right)\right)$ so that $p \in U \subset M$ and $x^{j}(p)=0, j=1,2$. Thus, around $p$, the second equation in (5) is simplified to

$$
D_{1} \phi \pm \mathrm{i} D_{2} \phi=0
$$

which says in view of the $\partial^{*}$-Poincare lemma (see [JT]) that, up to a nonvanishing factor, $\phi$ or $\phi^{*}$ is holomorphic around $p$. In particular, if $p$ is a zero of $\phi$, then locally,

$$
|\phi(x)|=|x|^{n} h(x),
$$


where $h>0$ and $n \geqq 1$ is an integer. The zero is obviously isolated. In this case, people say there is a string passing through $p$ with the winding number $n$. It is not hard to show that, for a nontrivial solution, $|\phi|<\varepsilon$ everywhere on $M$. Thus the third equation in (5) implies that the vorticity field acquires its maximal magnitude at the zeros of $\phi$. In other words, the zeros of $\phi$ give the locations of the vortices or strings of a solution.

Suppose the zeros of the Higgs field $\phi$ are labeled by $p_{1}, \ldots, p_{m} \in M$ with multiplicities $n_{1}, \ldots, n_{m}$, respectively. $N=n_{1}+\cdots+n_{m}$ is the total string or vortex number. The easily verified relation (cf. $[\mathrm{N} 1, \mathrm{~N} 2, \mathrm{Br}, \mathrm{Ga}]$ )

$$
N=\frac{1}{4 \pi}\left|\int_{M} \varepsilon^{j k} F_{j k} \mathrm{~d} \Omega_{g}\right|
$$

says that $N=\left|c_{1}(L)\right|$ (the first Chern number). Since $|\phi|$ has the local representation (12) around each point $p=p_{l}$ (with $n=n_{l}$ ), $l=1, \ldots, m$, we see that the substitution $u=\ln |\phi|^{2}$ renders the last two equations in (5) into the form

$$
\Delta_{g} u=\left(e^{u}-\varepsilon^{2}\right)+4 \pi \sum_{l=1}^{m} n_{l} \delta_{p_{l}}
$$

where $\delta_{p}$ is the Dirac distribution on $\left(M,\left\{g_{j k}\right\}\right)$ concentrated at $p$.

Using the last two equations in (5) and (10), we obtain

$$
\begin{aligned}
\pm \frac{1}{4} \varepsilon^{2} \varepsilon^{j k} F_{j k} \pm \nabla_{j}\left(\varepsilon^{j k} J_{k}\right) & =-\frac{1}{4} \varepsilon^{2}\left(|\phi|^{2}-\varepsilon^{2}\right)+\frac{1}{4} \Delta_{g}|\phi|^{2} \\
& =-\frac{1}{4} \varepsilon^{2}\left(e^{u}-\varepsilon^{2}\right)+\frac{1}{4} \Delta_{g} e^{u}
\end{aligned}
$$

Hence Eq. (11) becomes

$$
K_{g}=-\pi G\left(\varepsilon^{2}\left[e^{u}-\varepsilon^{2}\right]-\Delta_{g} e^{u}\right) .
$$

Let $u_{0}$ be a solution of the equation

$$
\Delta_{g} u_{0}=-\frac{4 \pi N}{|M|_{g}}+4 \pi \sum_{l=1}^{m} n_{l} \delta_{p_{l}},
$$

where $|M|_{g}$ is the total surface area of $\left(M,\left\{g_{j k}\right\}\right)$ (see [A]). Thus (13)-(14) may be put into the form

$$
\Delta_{g}\left(\varepsilon^{2} u-e^{u}-\varepsilon^{2} u_{0}\right)=-\frac{K_{g}}{\pi G}+\frac{4 \pi \varepsilon^{2} N}{|M|_{g}} .
$$

Since the function $\omega=\varepsilon^{2} u-e^{u}-\varepsilon^{2} u_{0}$ is smooth on $M$, the above equation leads to the consistency condition

$$
\frac{1}{\pi G_{M}} \int_{g} \mathrm{~d} \Omega_{g}=4 \pi \varepsilon^{2} N,
$$

where $\mathrm{d} \Omega_{g}$ is the canonical surface element of $M$ with respect to the metric $g=\left\{g_{j k}\right\}$. As a consequence of the well-known Gauss-Bonnet theorem, we are led from the relation (16) to Eq. (2). 
On the other hand, suppose that (2) is fulfilled. The existence part is a weaker version of the statement in Theorem 1.2 in the next subsection.

The theorem is proven.

Proof of the lower bound (4). We subtract (15) from (13) to obtain the equation

$$
\Delta_{g}\left(u-u_{0}\right)=e^{u}-\varepsilon^{2}+\frac{4 \pi N}{|M|_{g}} .
$$

Since $u-u_{0}$ and $e^{u}$ are smooth functions, integrating (17) yields the inequality

$$
4 \pi N<\varepsilon^{2}|M|_{g}
$$

Combining (2), (18) and using $\chi(M)=2$, we arrive at (4).

The Case of Symmetry-Restoration. Now set $\varepsilon=0$. We have seen that (1) and (5) are equivalent. Using the last equation in (5), we obtain

$$
4 \pi N= \pm \int_{M} \varepsilon^{j k} F_{j k} \mathrm{~d} \Omega_{g}=-\int_{M}|\phi|^{2} \mathrm{~d} \Omega_{g} .
$$

Thus $N=0$ and $\phi=0$. The 2-surface $M$ must be a flat torus, the energy density of the matter-gauge sector vanishes everywhere, and there is no gravity.

1.3. Existence of Multi-Strings. In the following, we state some existence results for $N$-string solutions over a compact Riemann surface $M$.

Theorem 1.2. Suppose that the integer $N$ satisfies the condition (2). For any $p_{1}, \ldots, p_{m} \in M$ and integers $n_{1}, \ldots, n_{m}$ with $n_{1}+\cdots+n_{m}=N$ and

$$
n_{1}<\frac{1}{2} N, \quad \cdots \cdots, \quad n_{m}<\frac{1}{2} N
$$

Eq. (1) or (5) have a smooth solution triplet $(g, \phi, A)$ so that it defines an appropriate Hermitian line bundle $L$ over $M$ with the first Chern number $c_{1}(L)=N$ and the zeros of the Higgs cross-section $\phi$ are exactly $p_{1}, \ldots, p_{m}$ with the respective multiplicities $n_{1}, \ldots, n_{m}$. In particular, when $N \geqq 3$, the system (1) or (5) has an $N$-string solution so that the Higgs field has simple zeros at $N$ distinct prescribed locations on $M$ if and only if the condition (2) is fulfilled.

The condition (19) is a technical restriction on the local string numbers. It is not clear at this moment whether it may be dropped. The existence theorem will be proved in Sect. 4 .

Remark. Our results may be compared with the interesting work [GOR] on Abelian global gravitational strings. There we find no-go theorems forbidding the existence of regular solutions without the gauge field coupling in a compact setting and asymptotically well-behaved solutions in a noncompact setting. Here, in the context of local strings with gauge field coupling, we have seen that there are values of the physical parameters which allow the existence of regular solutions in both settings (for existence results in the noncompact case, see [CHMcY] and Theorem 2.3 in the next section). 


\section{The Noncompact Case}

In this section, we study the cosmic string solutions of the coupled Einstein and Abelian Higgs equations (1) under the condition that the surface $M$ is noncompact and open. A typical case is that $M$ is globally conformal to the Euclidean plane $\mathbf{R}^{2}$. Thus we have $(M, g)=\left(\mathbf{R}^{2}, e^{\eta} \delta_{j k}\right)$. Two problems are considered. First, we will derive a class of obstructions of the form (2) to the existence of finite-energy string solutions under a radial symmetry assumption. Next, we obtain multi-string solutions realizing an arbitrarily prescribed string distribution and associated local string or vortex charges.

2.1. Obstructions to the Existence of Finite-Energy Solutions. We impose the following specific form for the unknown field configurations of solutions of Eqs. (1) which is standard in physics literature:

$$
\begin{aligned}
\eta(x) & =\eta(r) \\
\phi(x) & =U(r) e^{\mathrm{i} N \theta}, \\
A_{1}(x) & =-N V(r) \frac{x^{2}}{r^{2}}, \\
A_{2}(x) & =N V(r) \frac{x_{1}}{r^{2}},
\end{aligned}
$$

where $(r, \theta)$ are the polar coordinates of the point $x \in \mathbf{R}^{2}$ and the integer $N$ is the topological charge or the winding number.

Since the space is not compact, we do not expect to have an exact topological constraint like (2). Instead, we can state the following result.

Theorem 2.1. Under the cylindrical symmetry assumption (20) and the finiteenergy condition

$$
\int_{\mathbf{R}^{2}} \mathscr{E} e^{\eta} d x<\infty, \quad \int_{\mathbf{R}^{2}} K e^{\eta} d x<\infty,
$$

Eqs. (1) have a solution for $\varepsilon>0$ if and only if the topological charge $N$ satisfies the condition

$$
N<\frac{1}{\pi \varepsilon^{2} G} \text {. }
$$

In addition, the solution gives rise to a geodesically complete metric if and only if it fulfills the conditions stated in Theorem 2.3 below.

If $\varepsilon=0$, the system (1) has no solution except the trivial ones.

Our first recognition of an obstruction of the form (21) came through a study [CHMcY] of the self-dual reduction of (1), namely, the system (5) over $\left(\mathbf{R}^{2}, e^{\eta} \delta_{j k}\right)$, where $N=1 / 2 \pi \varepsilon^{2} G$ was recognized also as an obstruction. In this paper, we show that $N=1 / 2 \pi \varepsilon^{2} G$ is no longer an obstruction provided that the background metric is suitably chosen (see Theorem 2.3 below). In fact, that the existence at the critical case $N=1 / 2 \pi \varepsilon^{2} G$ eluded our detection in [CHMcY] was due to a special assumption on the background metric. There, we proved that no matter how the background metric is chosen, there is no finite-energy solution when (21) is violated. However, in the ranges

$$
0<N<\frac{1}{2 \pi \varepsilon^{2} G} \text { and } \frac{1}{2 \pi \varepsilon^{2} G}<N<\frac{1}{\pi \varepsilon^{2} G}
$$


there are finite-energy solutions with an arbitrary choice of the background metric (see Remark (b) in Sect. 6 for details). Therefore, in the radially symmetric case, the equality $N=1 / 2 \pi \varepsilon^{2} G$ is to be understood as an obstruction to the arbitrary choice of background metric. Here, to establish Theorem 2.1, we need only to show that (1) and (5) are equivalent under the symmetry assumption (20).

Theorem 2.2. The coupled Einstein and Abelian Higgs equations (1) and the selfdual system (5) are equivalent in the category of cylindrically symmetric solutions.

Remark. It is interesting to note that the equivalence assertion of Theorem 2.2 is valid for any cylindrically symmetric solutions, regardless of the asymptotic behavior. In particular, it says that for solutions of infinite energy, Eqs. (1) and (5) are also equivalent. Thus we can describe, as well, all infinite-energy solutions of (1) with the symmetry (20) by studying (5). In [CHMcY], we have shown that the equations (5) have regular entire solutions outside the region (21) for which the energy blows-ups take place at spatial infinity.

Proof of Theorem 2.2. The proof depends on the radial symmetry (20) because the asymptotic behavior of a solution triplet is not a priori known.

As in the last section, it suffices to show either $P^{+} \equiv 0$ or $P^{-} \equiv 0$. Suppose otherwise that $P^{-} \neq 0$. Then (7) says that $P^{+}=0$ somewhere. Using the radial ansatz (20), we see that there exists an $r \geqq 0$ so that

$$
P^{+}(r)=2 N e^{-\eta(r)} \frac{V_{r}(r)}{r}+\left(U^{2}(r)-\varepsilon^{2}\right)=0
$$

Set $Z=\left\{r \geqq 0 \mid P^{+}(r)=0\right\}$. We assert that the number of points in $Z, \# Z$, does not exceed 3 unless $P^{+} \equiv 0$. In fact, if $\# Z \geqq 4$ but $P^{+} \neq 0$, then there were $r_{1}, r_{2}, r_{3} \in Z$ so that $0<r_{1}<r_{2}<r_{3}$.

Inserting the radial ansatz $(20)$, we reduce (8) to the form

$$
P_{r r}^{+}+\frac{1}{r} P_{r}^{+} \geqq U^{2} e^{\eta} P^{+}
$$

Consider (22) over $\left(r_{1}, r_{3}\right)$. Since $P^{+}\left(r_{2}\right)=0, P^{+}$would have a nonnegative maximum inside the interval $\left(r_{1}, r_{3}\right)$, contradicting the strong maximum principle [GT]. Consequently $\# Z \leqq 3$. Hence $P^{+}(r) \neq 0$ for all $r>0$, with at most three exceptions. Therefore we must have $P^{-} \equiv 0$, another contradiction.

Assume now $P^{+} \neq 0$. Then (7) says that $P^{-}=0$ somewhere. The radial version of (9) reads

$$
P_{r r}^{-}+\frac{1}{r} P_{r}^{-} \leqq U^{2} e^{\eta} P^{-}
$$

Again, according to the strong maximum principle, $P^{-}$cannot have a nonpositive local minimum unless it is a constant. Thus we obtain $P^{-} \equiv 0$.

Proof of Theorem 2.1. If $\varepsilon>0$, it is convenient to introduce the rescaling

$$
x \mapsto \frac{x}{\varepsilon}, \quad \phi \mapsto \varepsilon \phi, \quad A_{j} \mapsto \varepsilon A_{j} .
$$


Then the energy density and Gaussian curvature obey the transformation rules

$$
\mathscr{E} \mapsto \varepsilon^{4} \mathscr{E}, \quad K_{g} \mapsto \varepsilon^{2} K_{g}
$$

in the new coordinates so that the Higgs potential function takes the normalized form

$$
\frac{1}{8}\left(|\phi|^{2}-1\right)^{2}
$$

As a consequence, the self-dual system (5) is written

$$
\begin{aligned}
K_{g}-4 \pi \varepsilon^{2} G \mathscr{E} & =0, \\
D_{j} \phi \pm \mathrm{i} \varepsilon_{j}^{k} D_{k} \phi & =0, \\
\varepsilon^{j k} F_{j k} \pm\left(|\phi|^{2}-1\right) & =0,
\end{aligned}
$$

which is the system studied in detail in [CHMcY]. It is known that a solution of (24) with the symmetry (20) carries finite energy if and only if (21) is satisfied. However, since by Theorem 2.2, Eqs. (1) and (5) are equivalent, we arrive at the first part of the conclusion.

The assertion on metric completeness is established in Sect. 6.

Assume now $\varepsilon=0$. Since Eqs. (1) and (5) are equivalent, we will use both of them to argue freely depending on convenience.

First, we observe that for a nontrivial solution of the form $(20)$, we have $U(r) \neq 0$ at any $r>0$. In fact, if there is an $r_{0}>0$ so that $U\left(r_{0}\right)=0$, then, applying the boundary condition $\phi=0$ on $|x|=r_{0}$ in the inequality $\Delta_{g}|\phi|^{2} \geqq|\phi|^{4}$ over $|x|<r_{0}$, we have $\phi=0$ for $|x|<r_{0}$. That is, $U(r)=0$ for $r<r_{0}$.

With the radial symmetry $(20)$, we have

$$
F_{12}=N \frac{V_{r}(r)}{r}
$$

Inserting this expression into the last equation in (5), i.e., $2 e^{\eta} F_{12} \pm U^{2}=0$, we obtain $V(r)=$ const. for $r<r_{0}$. However, the regularity of $A_{j}$ requires $V(0)=0$. Thus $V(r)=0$ for $r<r_{0}$. As a consequence, we find that $\mathscr{E}(\phi, A)(r)=0, r<r_{0}$. Using these facts in the radial version of (1), namely,

$$
\begin{aligned}
\eta_{r r}+\frac{1}{r} \eta_{r}+8 \pi G e^{\eta} \mathscr{E} & =0, \\
U_{r r}+\frac{1}{r} U_{r}-\frac{N^{2}}{r^{2}}(V-1)^{2} U-\frac{1}{2} e^{\eta} U^{3} & =0, \\
V_{r r}-\frac{1}{r}\left(1+r \eta_{r}\right) V_{r}-e^{\eta}(V-1) U^{2} & =0,
\end{aligned}
$$

and applying in (25) the uniqueness theorem for the initial value problem of ordinary differential equations, we conclude that $U(r)=0, V(r)=0$, and $\eta=$ const. for all $r>0$. Thus, we arrive at a trivial solution.

Consequently, we may assume in the following that $U(r) \neq 0$ for $r>0$. Since (25) is invariant under the reflection $U \mapsto-U$, we may assume also that $U(r)>0$, $r>0$. 
Suppose in (20), $N \geqq 1$. The regularity of $\phi$ at the origin requires that $U(0)=0$ and $U(r)=r^{N} f(r)$ near $r=0$, where $f(0) \neq 0$. Hence the substitution $u=\ln U^{2}$ reduces the last two equations in (5) to

$$
\begin{aligned}
& u_{r r}+\frac{1}{r} u_{r}=e^{\eta+u}, \quad r>0, \\
& \lim _{r \rightarrow 0} \frac{u(r)}{\ln r}=2 N .
\end{aligned}
$$

The boundary condition above implies $\lim _{r \rightarrow 0} r u_{r}(r)=2 N$. Thus

$$
r u_{r}(r)=2 N+\int_{0}^{r} \rho e^{\eta(\rho)+u(\rho)} \mathrm{d} \rho, \quad r>0 .
$$

In particular, $r u_{r}(r)>2 N, r>0$, which implies, say,

$$
u(r)>2 N \ln r+u(1), \quad r>1 .
$$

On the other hand, it is easy to calculate that $\left|D_{1} \phi\right|^{2}+\left|D_{2} \phi\right|^{2}=\frac{1}{2} u_{r}^{2} e^{u}$. Therefore we obtain the lower bound

$$
\left|D_{1} \phi\right|^{2}+\left|D_{2} \phi\right|^{2} \geqq \frac{1}{2} \cdot \frac{4 N^{2}}{r^{2}} \cdot r^{2 N} e^{u(1)}=2 N^{2} e^{u(1)} r^{2 N-2}, \quad|x|=r>1 .
$$

Consequently,

$$
\int_{\mathbf{R}^{2}} \mathscr{E} e^{\eta} \mathrm{d} x \geqq 4 \pi N^{2} e^{u(1)} \int_{1}^{\infty} r^{2 N-1} \mathrm{~d} r=\infty .
$$

In other words, we have energy blow-up.

If $N=0$, then by (20), $A_{j} \equiv 0, j=1,2$. Thus the last equation in (5) implies that $U \equiv 0$, contradicting the assumption that $U(r)>0, r>0$.

In conclusion, there is no nontrivial solution when $\varepsilon=0$ except those carrying infinite energy. The theorem is proven.

In the compact case, the nonexistence result at $\varepsilon=0$ may also be established quickly by a similar method. In fact, from the second equation in (1), we have as before $\Delta_{g}|\phi|^{2} \geqq|\phi|^{4}$. Thus the maximum principle implies that $\phi=0$ everywhere. Inserting this fact into the last equation in (5), we find $F_{j k}=0$. Thus $\mathscr{E}=0$ and $K_{g}=0$, which imply that $M$ is a 2-torus and the solution triplet $\left(\phi, A,\left\{g_{j k}\right\}\right)$ is trivial.

Remark. Taking $G=1$ and $\varepsilon>0$ sufficiently small, there are some earlier numerical studies of cosmic strings as static solutions of the coupled Einstein and Abelian Higgs equations. For example, we may cite [LM] in which numerical solutions are obtained for $\varepsilon$ lying in the range $10^{-2} \sim 10^{-1}$ and $N=1$. It is seen that these solutions are consistent with our obstruction (21). Moreover, (21) also allows us to point out that, whenever $\varepsilon \geqq 1 / \sqrt{\pi}$ (with $\lambda=1$ and $G=1$ ), finite-energy solutions will fail to exist. It seems to be a reasonable conjecture that similar obstructions are present even when the coupling parameter $\lambda \neq 1$.

2.2. An Existence Theorem for Multi-String Solutions. In Theorem 1.2, it is seen that we can construct multi-string solutions for (1) on a compact Riemann surface. The sufficiency condition stated in Theorem 1.2 imposes some restrictions to the 
local winding numbers $n_{l}$ 's of the strings. Such a barrier comes from the topological type of the base space and from our method in the existence proof (see Sect. 4). When $(M, g)$ is conformally flat, the constraint from the topology disappears and we are able to get solutions which realize an arbitrarily prescribed string distribution and the respectively designated local winding numbers $n_{l}$ 's. The condition for existence is only stated for the total charge $N$.

Here is our main existence result for the noncompact case.

Theorem 2.3. Consider the coupled Einstein and Abelian Higgs equations (1) over an open Riemann surface $(M, g)$. For any $p_{1}, \ldots, p_{m} \in M$ and $n_{1}, \ldots, n_{m} \in \mathbf{N}$, the system (1) has a finite-energy solution so that $M=\mathbf{R}^{2}, g$ is conformal to the standard metric of $\mathbf{R}^{2}: g_{j k}=e^{\eta} \delta_{j k}$, the zeros of $\phi$ are exactly $p_{1}, \ldots, p_{m}$ with the corresponding multiplicities $n_{1}, \ldots, n_{m}$, the conformal factor $e^{\eta}$ verifies the sharp decay estimate

$$
e^{\eta(x)}=O\left(|x|^{-4 \pi \varepsilon^{2} G N}\right) \text { as }|x| \rightarrow \infty
$$

and there hold the following quantized values of the total gravitational curvature, the magnetic flux, and the energy of the matter-gauge sector

$$
\int_{\mathbf{R}^{2}} K_{g} e^{\eta} d x=4 \pi^{2} \varepsilon^{2} G N, \quad \int_{\mathbf{R}^{2}} F_{12} d x=2 \pi N, \quad \int_{\mathbf{R}^{2}} \mathscr{E} e^{\eta} d x=\pi \varepsilon^{2} N,
$$

provided that the total string number $N=n_{1}+\cdots+n_{m}$ satisfies the bound

$$
N \leqq \frac{1}{2 \pi \varepsilon^{2} G} .
$$

Besides, the Gauss curvature $K_{g}$, and the physical energy terms $F_{j k}^{2},\left|D_{j} \phi\right|^{2}$, $\left(|\phi|^{2}-\varepsilon^{2}\right)^{2}$ obey the sharp decay estimates

$$
K_{g}, \quad F_{j k}^{2}, \quad\left|D_{j} \phi\right|^{2}, \quad 0<\varepsilon^{2}-|\phi|^{2}=O\left(|x|^{-b}\right) \quad \text { as }|x| \rightarrow \infty
$$

for any $b>0$ if $N<1 / 2 \pi \varepsilon^{2} G$. When $N=1 / 2 \pi \varepsilon^{2} G$ but $m \geqq 2$, there hold instead the asymptotic decay rates at $r=|x|=\infty$ :

$$
\begin{aligned}
K_{g} & =O\left(r^{-1}\right), & F_{j k}=O\left(r^{-4}\right), \\
\left|D_{j} \phi\right|^{2} & =O\left(r^{-3}\right), & 0<\varepsilon^{2}-|\phi|^{2}=O\left(r^{-2}\right) .
\end{aligned}
$$

While, when $N=1 / 2 \pi \varepsilon^{2} G$ and $m=1$, the radially symmetric solution satisfies

$$
\begin{aligned}
K_{g} & =O\left(r^{-2 \sqrt{2 N}}\right), \quad F_{j k}=O\left(r^{-(2+\sqrt{2 N})}\right), \\
\left|D_{j} \phi\right| & =O\left(r^{-(1+\sqrt{2 N})}\right), \quad 0<\varepsilon^{2}-|\phi|^{2}=O\left(r^{-\sqrt{2 N}}\right) .
\end{aligned}
$$

Furthermore, in the same category of solutions, the obtained surface $\left(\mathbf{R}^{2}, e^{\eta} \delta_{j k}\right)$ is geodesically complete if and only if the integer $N$ fulfills the above condition, $N \leqq 1 / 2 \pi \varepsilon^{2} G$. When $N>1 / 2 \pi \varepsilon^{2} G$, although there is an $N$-string solution and the corresponding Gauss curvature is the curvature function for some conformal metric which is complete, the obtained gravitational metric itself in the solution is not complete. 
Remarks. (a) We see that the condition $N \leqq 1 / 2 \pi \varepsilon^{2} G$ for existence of multi-string solutions is fairly general because $N>1 / 2 \pi \varepsilon^{2} G$ already leads to metric incompleteness. It is not clear whether there are finite-energy multi-string solutions in the range $1 / 2 \pi \varepsilon^{2} G<N<1 / \pi \varepsilon^{2} G$, but it seems clear that metric incompleteness is an early signal for energy blow-ups because we know that such a scenario is true in the context of radially symmetric solutions for which the strings are superimposed at one point [CHMcY]. Indeed it is not clear as to whether, after all, a similar obstruction as (21) is present for nonradial solutions of finite energies. Our analysis in Sect. 5 seems to indicate the existence of an obstruction. However, we were unable to establish it rigorously.

(b) The solutions stated in Theorem 2.3 are most interesting because they belong to the category that the Higgs field tends to the asymmetric vacuum at infinity in spite of the decay of the gravitational metric and are crucial in the Higgs mechanism. In the radially symmetric case, we have found that there are exactly two distinct families of solutions: those who go to the asymmetric vacuum (characterized by $|\phi|=1$ ) and those who go to the symmetric vacuum (characterized by $\phi=0$ ) at infinity. See [CHMcY] for details. There the first family of solutions was rather elusive to catch from the shooting data. In Sect. 6, we will show that the metrics arising from the second family of solutions are all incomplete. Thus Theorem 2.3 says that another interesting aspect of the solutions in the first family is that some of them (namely those in the range $N \leqq 1 / 2 \pi \varepsilon^{2} G$ ) can provide complete metrics.

In Sect. 5, we prove the existence of multi-strings for a given string distribution and obtain some preliminary properties of the solutions which will be useful in deriving the desired asymptotic estimates. In Sect. 6, we establish the decay estimates and identify in terms of the total string number $N$ the criterion for completeness of the gravitational metric.

\section{The Role of Defects}

When the gravitational sector is ignored and the Abelian Higgs model is considered in a two-dimensional framework, the equations govern the electromagnetic properties of a planar superconductor so that the Higgs field $\phi$ appears as an order parameter. At the places where $\phi$ vanishes, superconductivity is destroyed and the magnetic field penetrates the sample in the form of vortex-lines. Thus the zeros of $\phi$ are also called defects which are indicators of spots of partial restoration of the symmetric normal phase. It is already well-known in the Abelian Higgs model that the appearance of these defects is equivalent to the existence of mixed states characterized by $|\phi| \neq 1, F_{j k} \neq 0[\mathrm{Ab}, \mathrm{JT}]$. Here we would like to know whether the same statement holds in the presence of gravity, i.e., whether the existence of strings or defects is crucial to producing gravity or a nonflat spacetime. For technical reasons, we will mostly concentrate on the compact case. The general situation when the Higgs potential takes the form $\frac{\lambda}{8}\left(|\phi|^{2}-1\right)^{2}$ and $\lambda>0$ is arbitrary will be studied.

The basic result in this section says that the absence of string defects implies the absence of gravity. Such a fact may be seen intuitively as follows. When there are no string defects, the state is purely superconducting and the energy vacuum is attained. Thus the energy distribution of the matter-gauge sector is everywhere zero. However, the Einstein equations (i.e., the first equation in (1)) imply that the 
space curvature vanishes identically. As a consequence, we arrive at a flat space with trivial topology and there is no gravity.

With the notation in Sect. 1, the Einstein and Abelian Higgs equations become

$$
\begin{aligned}
K_{g} & =4 \pi G \mathscr{E}, \quad T_{j k}=0, \\
\frac{1}{\sqrt{g}} D_{j}\left(g^{j k} \sqrt{g}\left[D_{k} \phi\right]\right) & =\frac{\lambda}{2}\left(|\phi|^{2}-\varepsilon^{2}\right) \phi, \quad x \in M . \\
\frac{1}{\sqrt{g}} \partial_{j^{\prime}}\left(g^{j k} g^{j^{\prime} k^{\prime}} \sqrt{g} F_{k k^{\prime}}\right) & =\frac{\mathrm{i}}{2} g^{j k}\left(\phi\left[D_{k} \phi\right]^{*}-\phi^{*}\left[D_{k} \phi\right]\right),
\end{aligned}
$$

Suppose that there are no defects, i.e., $\phi(x) \neq 0, \forall x \in M$. Then the line bundle $L$ over $M$ defined by the solution of (26) is trivial: $L=M \times \mathbf{C}$. We may view $\phi$ as a complex-valued function on $M$. Thus there is a real-valued function $f \in C^{\infty}(M)$ so that $\phi=\varphi e^{\mathrm{i} f}$ where $\varphi=|\phi|>0$. Perform the gauge transformation

$$
\phi \mapsto \phi e^{-\mathrm{i} f}, \quad A_{j} \mapsto A_{j}-\partial_{j} f
$$

in (26). We see that the last two equations in (26) take the form

$$
\begin{aligned}
\Delta_{g} \varphi & =\left(g^{j k} A_{j} A_{k}\right) \varphi+\frac{\lambda}{2}\left(\varphi^{2}-\varepsilon^{2}\right) \varphi, \\
\frac{1}{\sqrt{g}} \partial_{j}\left(g^{j k} \sqrt{g} A_{k}\right) & =-2 g^{j k} A_{j} \partial_{k} \ln \varphi \\
\frac{1}{\sqrt{g}} \partial_{j^{\prime}}\left(g^{j k} g^{j^{\prime} k^{\prime}} \sqrt{g} F_{k k^{\prime}}\right) & =-g^{j k} A_{k} \varphi^{2} .
\end{aligned}
$$

From the third equation in (27), we obtain

$$
\frac{1}{\sqrt{g}} \partial_{j^{\prime}}\left(g^{j^{\prime} k^{\prime}} \sqrt{g}\left[g^{j k} A_{j} F_{k k^{\prime}}\right]\right)+\frac{1}{2} g^{j^{\prime} k^{\prime}} g^{j k} F_{j j^{\prime}} F_{k k^{\prime}}=-g^{j k} A_{j} A_{k} \varphi^{2} .
$$

However, the first term on the left-hand side of (28) is a total divergence, thus an integration of (28) leads to

$$
\int_{M}\left\{\frac{1}{2} g^{j j^{\prime}} g^{k k^{\prime}} F_{j k} F_{j^{\prime} k^{\prime}}+g^{j k} A_{j} A_{k} \varphi^{2}\right\} \mathrm{d} \Omega_{g}=0 .
$$

The two terms in the integrand are both positive-definite. As a consequence, we obtain $A_{J}=0, j=1,2$. Inserting this information into the first equation in (27), we have

$$
\Delta_{g}(\varphi-\varepsilon)=\frac{\lambda}{2} \varphi(\varphi+\varepsilon)(\varphi-\varepsilon), \quad x \in M .
$$

Using $\varphi>0$ and the maximum principle in (29), we find $\varphi \equiv \varepsilon$. Consequently, $\mathscr{E}=0$ everywhere. In view of the first equation in (26), we arrive at $K_{g} \equiv 0$, which indicates that $M$ is a flat torus, gravity is absent, and the solution is trivial.

We summarize the above discussion as follows.

Theorem 3.1. For a solution triplet $\left(\phi, A,\left\{g_{j k}\right\}\right)$ of the general Einstein and Abelian Higgs equations (24) with $\lambda>0$ and $M$ being a compact 2-surface, the 
absence of string-like topological defects, i.e., $\phi \neq 0$ on $M$, implies that the solution is gauge-equivalent to a trivial solution so that $M$ must be a flat torus, which is characterized by $K_{g}=0, \phi=\varepsilon, A=0$.

A similar study may be carried out for the noncompact case as far as the field configurations decay fast enough at infinity so that the boundary terms resulting from integrating the first term on the left-hand side of (28) drop off. Thus, if such a property holds, the statement of Theorem 3.1 is also valid in general.

\section{Proof of Theorem 1.2}

Suppose that we can solve the coupled system (13)-(14) for the unknown metric $g$ and function $u$. Using $u$ and applying the methods in [N1, N2, Br, Ga], we can construct a suitable line bundle $L$ over $(M, g)$ equipped with a Hermitian metric so that $c_{1}(L)=N$ and a solution pair $(\phi, A)$ of the last two equations in (5) is obtained. The validity of the first equation in (5) follows directly from (14). Thus we may only concentrate on the solvability of (13)-(14).

Our main strategy is as follows. We first combine the two equations, (13) and (14), into a single equation with a peculiar-looking nonlinearity. Then we perturb the resulting equation by a positive parameter so that a supersolution may be constructed in such a way that a monotone iterative scheme can be used to find a classical solution. Finally we take the zero parameter limit to recover a solution of the original equation.

4.1. Reduction to an Elliptic Equation. We shall use the following standard device to get rid of the unknown gravitational background metric $g$ on $M$.

Assume that $g$ is conformal to a known metric $g_{0}$ :

$$
g=e^{\eta} g_{0}
$$

where $\eta$ is an unknown conformal exponent, and that $K_{g_{0}}$ is the Gauss curvature of $\left(M, g_{0}\right)$. Then $\eta, K_{g}, K_{g_{0}}$ are related through the equation

$$
-\Delta_{g_{0}} \eta+2 K_{g_{0}}=2 K_{g} e^{\eta} .
$$

Besides, we have $\Delta_{g}=e^{-\eta} \Delta_{g_{0}}$. Hence Eqs. (13)-(14) become

$$
\begin{gathered}
\Delta_{g_{0}} u=e^{\eta}\left(e^{u}-\varepsilon^{2}\right)+4 \pi \sum_{l=1}^{m} n_{l} \delta_{p_{l}}, \\
\Delta_{g_{0}}\left(-\eta-2 \pi G e^{u}\right)=-2 K_{g_{0}}-2 \pi G \varepsilon^{2} e^{\eta}\left(e^{u}-\varepsilon^{2}\right),
\end{gathered}
$$

where now $\delta_{p}$ is the Dirac distribution at $p$ on $\left(M, g_{0}\right)$.

Let $v=u-u_{0}$ where $u_{0}$ is a solution of

$$
\Delta_{g_{0}} u_{0}=-\frac{4 \pi N}{|M|_{g_{0}}}+4 \pi \sum_{l=1}^{m} n_{l} \delta_{p_{l}}
$$

Then (30)-(31) take the form

$$
\Delta_{g_{0}} v=e^{\eta}\left(e^{u_{0}+v}-\varepsilon^{2}\right)+\frac{4 \pi N}{|M|_{g_{0}}},
$$




$$
\Delta_{g_{0}}\left(-\eta-2 \pi G e^{u_{0}+v}+2 \pi G \varepsilon^{2} v\right)=-2 K_{g_{0}}+\frac{8 \pi^{2} G \varepsilon^{2} N}{|M|_{g_{0}}} .
$$

Using (2) and the Gauss-Bonnet theorem, we see that the right-hand side of (34) has zero integral. Consequently, there is a smooth function $v_{0}$ on $M$ so that

$$
\eta+2 \pi G e^{u_{0}+v}-2 \pi G \varepsilon^{2} v=v_{0}+c,
$$

where $c$ is an arbitrary constant to be adjusted later.

We now insert (35) into (33). Use the notation $a=2 \pi G$. Then we arrive at the following equation,

$$
\Delta_{g_{0}} v=\lambda e^{v_{0}+a \varepsilon^{2} v-a e^{u_{0}+v}}\left(e^{u_{0}+v}-\varepsilon^{2}\right)+\frac{4 \pi N}{|M|_{g_{0}}} \text { on } M .
$$

Note that $\lambda=e^{c}$ is an adjustable parameter which should not be confused with the coupling parameter $\lambda$ in the previous sections. We shall find in the rest of this section a solution of (36) which is obviously equivalent to (30)-(31) or (33)-(34).

4.2. The Perturbed Problem. Let $\left(U_{l},\left(x^{j}\right)\right)$ be an isothermal coordinate chart near $p_{l} \in M$ for the surface $\left(M, g_{0}\right)$ so that $x^{j}\left(p_{l}\right)=0(j=1,2)$. According to [A], when $U_{l}$ is small the function $u_{0}$ (see (32)) has the property

$$
u_{0}(x)=n_{l} \ln |x|^{2}+w_{l}(x) \text { in }\left(U_{l},\left(x^{j}\right)\right)
$$

where $w_{l}$ is a smooth function on $U_{l}$.

We can use (37) to define a regular perturbation of $u_{0}$ as follows.

For any $\sigma>0$ so that $p \in U_{l}$ whenever $|x(p)|<3 \sigma$, choose a function $\rho \in C^{\infty}(M)$ satisfying

$$
0 \leqq \rho \leqq 1, \quad \rho(p)=1 \quad \text { for }|x(p)|<\sigma, \quad \rho(p)=0 \text { for }|x(p)|>2 \sigma .
$$

Take $\sigma$ sufficiently small so that

$$
u_{0}^{\delta}(x)=n_{l} \ln \left(|x|^{2}+\delta \rho(x)\right)+w_{l}(x) \quad \text { in }\left(U_{l},\left(x^{j}\right)\right), \quad l=1, \ldots, m
$$

$(\delta>0)$ naturally extends to a smooth function on the full $M$ and

$$
u_{0}^{\delta}=u_{0} \quad \text { in } M-\bigcup_{l=1}^{m} U_{l} ; \quad u_{0} \leqq u_{0}^{\delta} \quad \text { in } M .
$$

It is more transparent to rewrite (36) in the form

$$
\Delta_{g_{0}} v=\lambda e^{v_{0}-a \varepsilon^{2} u_{0}+a \varepsilon^{2}\left(u_{0}+v\right)-a e^{u_{0}+v}}\left(e^{u_{0}+v}-\varepsilon^{2}\right)+\frac{4 \pi N}{|M|_{g_{0}}} \text { on } M .
$$

The function $e^{-a \varepsilon^{2} u_{0}}$ is a singular factor. We overcome this difficulty by introducing the perturbed equation

$$
\Delta_{g_{0}} v=\lambda e^{v_{0}-a \varepsilon^{2} u_{0}^{\delta}+a \varepsilon^{2}\left(u_{0}+v\right)-a e^{u_{0}+v}}\left(e^{u_{0}+v}-\varepsilon^{2}\right)+\frac{4 \pi N}{|M|_{g_{0}}} \text { on } M .
$$

From now on, we assume $0<\delta<1$ (say). 


\subsection{The Sub/Supersolution}

Lemma 4.1. There is a smooth function $w$ on $M$ independent of $\delta$ so that

$$
\Delta_{g_{0}} w>\lambda e^{v_{0}-a \varepsilon^{2} u_{0}^{\delta}+a \varepsilon^{2}\left(u_{0}+w\right)-a e^{u_{0}+w}}\left(e^{u_{0}+w}-\varepsilon^{2}\right)+\frac{4 \pi N}{|M|_{g_{0}}} \text { on } M .
$$

for some suitable $\lambda$. Namely $w$ is a subsolution of (40) for all $\delta$.

Proof. As before, let $\left(U_{l},\left(x^{j}\right)\right)$ be a coordinate chart near $p_{l} \in M$. Suppose that $\sigma>0$ is a small number so that

$$
\left\{x \in \mathbf{R}^{2}|| x \mid<3 \sigma\right\} \subset\left\{x \in \mathbf{R}^{2} \mid x=x(p) \text { for some } p \in U_{l}\right\}, l=1, \ldots, m .
$$

Define a function $f_{\sigma} \in C^{\infty}(M)$ so that

$$
\begin{gathered}
0 \leqq f_{\sigma} \leqq 1, \quad f_{\sigma}(p)=1 \quad \text { for }|x(p)| \leqq \sigma \text { and } p \in U_{l}, \quad l=1, \ldots, m, \\
f_{\sigma}(p)=0 \text { for }|x(p)| \geqq 2 \sigma \text { and } \quad p \in U_{l}, \quad l=1, \ldots, m, \quad \text { or } p \in M-\cup_{l}^{m} U_{l} .
\end{gathered}
$$

Then the equation

$$
\Delta_{g_{0}} w=\frac{8 \pi N}{|M|_{g_{0}}} f_{\sigma}-C(\sigma)
$$

has a solution if $C(\sigma)$ satisfies

$$
C(\sigma)=\frac{8 \pi N}{|M|_{g_{0}}^{2}} \int_{M} f_{\sigma} \mathrm{d} \Omega_{g_{0}}
$$

The solution is unique up to an additive constant.

By (43) and the definition of $f_{\sigma}$, we see that $C(\sigma) \rightarrow 0$ as $\sigma \rightarrow 0$. Thus we can choose suitable $\sigma>0$ to make

$$
\frac{8 \pi N}{|M|_{g_{0}}^{2}}-C(\sigma)>\frac{4 \pi N}{|M|_{g_{0}}^{2}}
$$

Define

$$
U_{l}^{\sigma}=\left\{p \in M\left|p \in U_{l},\right| x(p) \mid<\sigma\right\}, \quad l=1, \ldots, m .
$$

Hence, in view of (42) and (44), we have

$$
\Delta_{g_{0}} w>\frac{4 \pi N}{|M|_{g_{0}}^{2}} \text { in } \bigcup_{l=1}^{m} U_{l}^{\sigma}
$$

Of course, we can choose $w$ such that $e^{u_{0}+w}-\varepsilon^{2}<0$ on $M$. Consequently the inequality (41) holds in $\cup_{l=1}^{m} U_{l}$ for any $\lambda, \delta$.

Recall that $w$ is independent of $\delta$. Besides, by the definition of $u_{0}^{\delta}$, we have $u_{0}^{\delta} \leqq u_{0}^{1}$. Thus

$$
\begin{aligned}
e^{-a \varepsilon^{2} u_{0}^{\delta}+a \varepsilon^{2}\left(u_{0}+w\right)-a e^{u_{0}+w}} & \geqq e^{a \varepsilon^{2}\left(u_{0}-u_{0}^{1}\right)+a \varepsilon^{2} w-a e^{u_{0}+w}} \\
& \geqq C_{0}>0 \quad \text { in } M-\bigcup_{l=1}^{m} U_{l}^{\sigma} .
\end{aligned}
$$


Of course,

$$
\sup _{M}\left(e^{u_{0}+w}-\varepsilon^{2}\right)=-C_{1}, \quad C_{1}>0 .
$$

The constants $C_{0}, C_{1}$ are independent of $\delta$. From (45)-(46), we see that when $\lambda$ is large enough, the inequality (41) holds in $M-\cup_{l=1}^{m} U_{l}^{\sigma}$ as well.

In summary, $w$ satisfies (41) for all $\delta$.

Lemma 4.2. Define $v_{1}=-u_{0}+\ln \varepsilon^{2}$. Then $v_{1}>w$ on $M$.

Proof. Use the notation of Lemma 4.1. Suppose $\sigma>0$ is small so that $U_{l}^{\sigma} \cap U_{l^{\prime}}^{\sigma}=\emptyset$ for $l \neq l^{\prime}$. Of course,

$$
w<v_{1} \quad \text { in } \bar{U}_{l}^{\sigma}, \quad l=1, \ldots, m
$$

when $\sigma$ is sufficiently small. Using (32), we can rewrite (41) in the form

$$
\begin{gathered}
\Delta_{g_{0}}\left(u_{0}+w-\ln \varepsilon^{2}\right)>\lambda \varepsilon^{2} e^{v_{0}-a \varepsilon^{2} u_{0}^{\delta}+a \varepsilon^{2}\left(u_{0}+w\right)-a e^{u_{0}+w}}\left(e^{u_{0}+w-\ln \varepsilon^{2}}-1\right) \\
\text { in } M-\bigcup_{l=1}^{m} U_{l}^{\sigma} .
\end{gathered}
$$

On $\partial U_{l}^{\sigma}(l=1, \ldots, m)$, we already have $u_{0}+w-\ln \varepsilon^{2}<0$. If there is a point $p$ so that $\left(u_{0}+w-\ln \varepsilon^{2}\right)(p) \geqq 0$, then the function $u_{0}+w-\ln \varepsilon^{2}$ has a nonnegative interior maximum in $M-\cup_{l=1}^{m} U_{l}^{\sigma}$ which is false due to (47) and the maximum principle. The lemma is proven.

4.4. Solution of the Perturbed Equation. We shall use $v_{1}$ as a supersolution to find a solution of (40). Note that $v_{1}$ is singular at the points $p_{1}, \ldots, p_{m}$. Nevertheless, we can apply the following iterative scheme. We define

$$
\begin{aligned}
\left(\Delta_{g_{0}}-C_{\delta}\right) v_{n}= & \lambda e^{v_{0}-a \varepsilon^{2} u_{0}^{\delta}+a \varepsilon^{2}\left(u_{0}+v_{n-1}\right)-a e^{u_{0}+v_{n-1}}}\left(e^{u_{0}+v_{n-1}}-\varepsilon^{2}\right) \\
& -C_{\delta} v_{n-1}+\frac{4 \pi N}{|M|_{g_{0}}} \text { on } M, \quad n=2,3, \ldots,
\end{aligned}
$$

where $C_{\delta}>0$ is a constant to be determined as follows.

Consider the function

$$
f(t)=e^{a \varepsilon^{2} t-a e^{t}}\left(e^{t}-\varepsilon^{2}\right) .
$$

It is direct to see that the derivative $f^{\prime}(t)$ is bounded for $t \in \mathbf{R}$.

Set

$$
C_{\delta}=1+\lambda \sup _{x \in M}\left\{e^{v_{0}(x)-a \varepsilon^{2} u_{0}^{\delta}(x)}\right\} \cdot \sup _{t \in \mathbf{R}}\left\{f^{\prime}(t)\right\}
$$

in (48). We have

Lemma 4.3. There holds on $M$ the inequality

$$
v_{1}>v_{2}>\cdots>v_{n}>\cdots>w .
$$

Proof. We have already seen in Lemma 4.2 that $v_{1}>w$. Using (48), we obtain

$$
\left(\Delta_{g_{0}}-C_{\delta}\right) v_{2}=-C_{\delta} v_{1}+\frac{4 \pi N}{|M|_{g_{0}}} \text { on } M .
$$


Thus $\left(\Delta_{g_{0}}-C_{\delta}\right)\left(v_{2}-v_{1}\right)=0$ in $M-\left\{p_{1}, \ldots, p_{m}\right\}$. Since $v_{1} \in L^{p}(M)$ for any $p>1$, we have $v_{2} \in W^{2, p}(M)$ and $v_{2} \in C^{1, \alpha}(M)$ for any $0<\alpha<1$. In particular $v_{2}$ is bounded. Using the maximum principle, we get $v_{1}>v_{2}$.

Besides, using the notation (49), the inequality (41), i.e.,

$$
\Delta_{g_{0}} w>\lambda e^{v_{0}-a \varepsilon^{2} u_{0}^{\delta}} f\left(u_{0}+w\right)+\frac{4 \pi N}{|M|_{g_{0}}},
$$

Lemma 4.2, and (51), we find

$$
\begin{aligned}
\left(\Delta_{g_{0}}-C_{\delta}\right)\left(w-v_{2}\right) & >\lambda e^{v_{0}-a \varepsilon^{2} u_{0}^{\delta}}\left(f\left(u_{0}+w\right)-f\left(u_{0}+v_{1}\right)\right)-C_{\delta}\left(w-v_{1}\right) \\
& =\left(\lambda e^{v_{0}-a \varepsilon^{2} u_{0}^{\delta}} f^{\prime}\left(u_{0}+\xi\right)-C_{\delta}\right)\left(w-v_{1}\right) \\
& >0 \quad\left(w<\xi<v_{1}\right) .
\end{aligned}
$$

Thus the maximum principle implies that $w<v_{2}$.

Suppose $v_{k-1}>v_{k}>w$ with $k \geqq 2$. Then (48) says that

$$
\begin{aligned}
\left(\Delta_{g_{0}}-C_{\delta}\right)\left(v_{k+1}-v_{k}\right) & =\lambda e^{v_{0}-a \varepsilon^{2} u_{0}^{\delta}}\left(f\left(u_{0}+v_{k}\right)-f\left(u_{0}+v_{k-1}\right)\right)-C_{\delta}\left(v_{k}-v_{k-1}\right) \\
& =\left(\lambda e^{v_{0}-a \varepsilon^{2} u_{0}^{\delta}} f^{\prime}\left(u_{0}+\xi_{k}\right)-C_{\delta}\right)\left(v_{k}-v_{k-1}\right)>0,
\end{aligned}
$$

where $\xi_{k}$ is between $v_{k-1}$ and $v_{k}$. Hence $v_{k+1}<v_{k}$.

Moreover, we have

$$
\begin{aligned}
\left(\Delta_{g_{0}}-C_{\delta}\right)\left(w-v_{k+1}\right) & >\lambda e^{v_{0}-a \varepsilon^{2} u_{0}^{\delta}}\left(f\left(u_{0}+w\right)-f\left(u_{0}+v_{k}\right)\right)-C_{\delta}\left(w-v_{k}\right) \\
& =\left(\lambda e^{v_{0}-a \varepsilon^{2} u_{0}^{\delta}} f^{\prime}\left(u_{0}+\xi_{k}\right)-C_{\delta}\right)\left(w-v_{k}\right)>0 .
\end{aligned}
$$

Consequently $w<v_{k+1}$. The proof of the lemma is finished.

Taking the limit

$$
\lim _{n \rightarrow \infty} v_{n} \equiv v^{\delta}
$$

in (48) and using (50), we see that $v^{\delta}$ is a solution of Eq. (40) satisfying

$$
v_{1}>v^{\delta} \geqq w \quad \text { on } M \text {. }
$$

In order to find a solution of the original equation (39) or (35), we need to consider the $\delta \rightarrow 0$ limit in the following subsection.

4.5. Passage to the Limit $\delta \rightarrow 0$. First, observe that (52) implies that, for any $p>1$, there is a constant $C>0$ independent of $\delta$ so that

$$
\left\|v^{\delta}\right\|_{L^{p}(M)} \leqq C .
$$

Next, it is easy to see that the one-parameter function $f\left(u_{0}+v^{\delta}\right)(x)$ is uniformly bounded.

Besides, since $e^{-a \varepsilon^{2} u_{0}^{\delta}} \leqq e^{-a \varepsilon^{2} u_{0}}$ and in the local isothermal coordinates $\left(U_{l},\left(x^{j}\right)\right)$ 
around $p_{l}$ we have

$$
e^{-a \varepsilon^{2} u_{0}(x)}=e^{-a \varepsilon^{2} w_{l}(x)}|x|^{-2 a \varepsilon^{2} n_{l}}
$$

due to (37), we see that there is a constant $C>0$ independent of $\delta$ so that

$$
\left\|e^{-a \varepsilon^{2} u_{0}^{\delta}}\right\|_{L^{p}(M)} \leqq C,
$$

provided that

$$
2 a \varepsilon^{2} n_{l} p<2 .
$$

From the condition (2) and $\chi(M)=2$, we have

$$
a \varepsilon^{2} N=2 \text {. }
$$

Combining (55)-(56), we arrive at the condition

$$
n_{l} p<\frac{1}{2} N, \quad l=1, \ldots, m .
$$

Suppose that $n_{l}$ 's satisfy the restriction (19). Then there is a $p>1$ so that (57) is valid. Using (53)-(54) in (40), we see that there is a constant $C>0$ independent of $\delta$ to confine the $W^{2, p}$-norm of $v^{\delta}$ :

$$
\left\|v^{\delta}\right\|_{W^{2, p}(M)} \leqq C, \quad \forall 0<\delta<1 .
$$

From the embedding $W^{k, p}(M) \rightarrow C^{m}(M)$ for $0 \leqq m<k-2 / p$ with $k=2$ and $p>1$, we infer in view of (58) that

$$
\left|v^{\delta}\right|_{C(M)} \leqq C
$$

for any $\delta$, where $C>0$ is a $\delta$-independent constant.

It is now useful to rewrite (40) with $v=v^{\delta}$ in the form

$$
\Delta_{g_{0}} v^{\delta}=\lambda e^{v_{0}+a \varepsilon^{2}\left(u_{0}-u_{0}^{\delta}\right)+a \varepsilon^{2} v^{\delta}-a e^{u_{0}+v^{\delta}}}\left(e^{u_{0}+v^{\delta}}-\varepsilon^{2}\right)+\frac{4 \pi N}{|M|_{g_{0}}} .
$$

By the definition of $u_{0}^{\delta}$, we know that the factor $e^{a \varepsilon^{2}\left(u_{0}-u_{0}^{\delta}\right)}$ is bounded. Using (59), we see in particular that the right-hand side of $(60)$ has uniformly bounded $L^{p}$ norm for any $p>1$. Thus (58) holds for any $p>1$. As a consequence, $\left\{v^{\delta}\right\}$ is bounded in $C^{1, \alpha}$ for any $0<\alpha<1$. Applying this fact in (60), we conclude that $\left\{v^{\delta}\right\}$ is bounded in $C^{2, \alpha}(M)$. However, the compact embedding $C^{2, \alpha}(M) \rightarrow C^{2}(M)$ enables us to get a convergent subsequence $\left\{v^{\delta_{n}}\right\}\left(\delta_{n} \rightarrow 0\right.$ as $\left.n \rightarrow \infty\right)$ so that

$$
v^{\delta_{n}} \rightarrow \text { some element } v \text { in } C^{2}(M) .
$$

Inserting (61) into (60), we find that $v$ is a solution of (36).

The proof of Theorem 1.2 is complete.

\section{Proof of Theorem 2.3: Existence Part}

We now study the Einstein-Bogomol'nyi system (24) on the full plane. We shall look for a solution of the system so that it is such that the Higgs field $\phi$ vanishes 
precisely at the given points $p_{1}, \ldots, p_{m} \in \mathbf{R}^{2}$ at the respective orders $n_{1}, \ldots, n_{m}$. We can use the same device to reduce this problem to a system of nonlinear elliptic equations of the form (30)-(31). Comparing (5) and (24), it is easy to see that the new system may be obtained by a rescaling of the parameters in (30)-(31):

$$
\varepsilon \mapsto 1, \quad 2 \pi G \mapsto 2 \pi G \varepsilon^{2} .
$$

This simplification is convenient for our discussion to follow.

5.1. The Elliptic Equation and its Perturbation. Set $a=2 \pi G \varepsilon^{2}$ as in Sect. 4. Choose $g_{0}$ to be the standard flat metric. Hence $\Delta_{g_{0}}=\Delta, K_{g_{0}}=0$, and we end up with the following equations on $\mathbf{R}^{2}$ :

$$
\begin{gathered}
\Delta u=e^{\eta}\left(e^{u}-1\right)+4 \pi \sum_{l=1}^{m} n_{l} \delta_{p_{l}}, \\
\Delta\left(\eta+a e^{u}\right)=a e^{\eta}\left(e^{u}-1\right) .
\end{gathered}
$$

Define the background functions

$$
\begin{aligned}
& u_{0}=\sum_{l=1}^{m} n_{l} \ln \left(\frac{\left|x-p_{l}\right|^{2}}{1+\left|x-p_{l}\right|^{2}}\right), \\
& w_{0}=\sum_{l=1}^{m} n_{l} \ln \left(1+\left|x-p_{l}\right|^{2}\right) .
\end{aligned}
$$

Then it is seen that $u_{0}<0, w_{0} \geqq 0$, and

$$
\begin{aligned}
\Delta u_{0} & =4 \pi \sum_{l=1}^{m} n_{l} \delta_{p_{l}}-\Delta w_{0} \\
& =4 \pi \sum_{l=1}^{m} n_{l} \delta_{p_{l}}-g,
\end{aligned}
$$

where, in this section and part of the next section,

$$
g=\Delta w_{0}=4 \sum_{l=1}^{m} \frac{n_{l}}{\left(1+\left|x-p_{l}\right|^{2}\right)^{2}}>0
$$

which should not be confused with our notation for the Riemannian metric $g=\left\{g_{j k}\right\}$.

Let $u=u_{0}+v$. Then (62) becomes

$$
\Delta v=e^{\eta}\left(e^{u_{0}+v}-1\right)+g .
$$

By (63)-(64), we easily infer that

$$
h=\frac{\eta}{a}+e^{u_{0}+v}-v+w_{0}
$$

is an entire harmonic function, which clearly defines a background for the gravitational metric. The choice of $h$ is often crucial in establishing existence in some 
situations. In the radially symmetric case, $h$ is a constant. We now take the point of view that, far away from local regions, the solutions look radially symmetric, thus, multi-string solutions and radially symmetric string solutions should reside in the same metric background. Therefore we are led to assuming that $h=c=$ const. Of course, such a choice may restrict the range of non-symmetric solutions we are searching for, and hence, is only a technical convenience. In this section, we again use the notation $\lambda=e^{a c}$ as in the compact case, which should not be confused with the coupling parameter $\lambda$ considered in Sects. $0-3$. Note that this parameter is adjustable as in the compact case. Finally, inserting the above expression into (64), we find the resulting equation

$$
\Delta v=\lambda e^{-a w_{0}+a\left(v-e^{u_{0}+v}\right)}\left(e^{u_{0}+v}-1\right)+g \text { in } \mathbf{R}^{2} .
$$

As in the last section, Eq. (65) is not convenient to work with. We can avoid the difficulty by introducing the following $\delta$-regularization of $(65)$ :

$$
\Delta v=\lambda e^{-a w_{0}+a\left(v-e^{u_{0}^{\delta}+v}\right)}\left(e^{u_{0}^{\delta}+v}-1\right)+g \text { in } \mathbf{R}^{2},
$$

where

$$
u_{0}^{\delta}=\sum_{l=1}^{m} n_{l} \ln \left(\delta+\left|x-p_{l}\right|^{2}\right)-w_{0}
$$

Note that

$$
\Delta u_{0}^{\delta}=4 \delta \sum_{l=1}^{m} \frac{n_{l}}{\left(\delta+\left|x-p_{l}\right|^{2}\right)^{2}}-g
$$

5.2. The Solution of the Perturbed Equation via Sub/Supersolutions. We first find a supersolution of (66).

Lemma 5.1. The function $v_{1}^{\delta}=-u_{0}^{\delta}(0<\delta<1)$ is a supersolution of (66).

Proof. By virtue of (67),

$$
\begin{aligned}
\Delta v_{1}^{\delta} & =-4 \delta \sum_{l=1}^{m} \frac{n_{l}}{\left(\delta+\left|x-p_{l}\right|^{2}\right)^{2}}+g \\
& <g=\lambda e^{-a w_{0}+a\left(v_{1}^{\delta}-e^{u_{0}^{\delta}+v_{1}^{\delta}}\right.}\left(e^{u_{0}^{\delta}+v_{1}^{\delta}}-1\right)+g .
\end{aligned}
$$

Thus $v_{1}^{\delta}$ is a supersolution as expected.

We now turn to the recognition of a suitable subsolution.

Lemma 5.2. There is a $\lambda_{0}$ independent of $0<\delta<1 / 2$ (say) so that for aN $\leqq 1$, there holds

$$
0>\lambda e^{-a w_{0}-a e^{u_{0}^{\delta}}}\left(e^{u_{0}^{\delta}}-1\right)+g \text { in } \mathbf{R}^{2}
$$

whenever $\lambda>\lambda_{0}$. In other words, $v=0$ is a subsolution of (66) for all $\delta$.

Proof. We rewrite $u_{0}^{\delta}$ as

$$
u_{0}^{\delta}=-\sum_{l=1}^{m} n_{l} \ln \left(1+\frac{1-\delta}{\delta+\left|x-p_{l}\right|^{2}}\right)<0
$$


and

$$
e^{u_{0}^{\delta}}-1=e^{\xi\left(u_{0}^{\delta}\right) u_{0}^{\delta}} u_{0}^{\delta} \quad\left(0<\xi\left(u_{0}^{\delta}\right)<1\right) .
$$

Note that, since $u_{0}^{\delta}<0$,

$$
e^{\xi\left(u_{0}^{\delta}\right) u_{0}^{\delta}}=\frac{e^{u_{0}^{\delta}-1}}{u_{0}^{\delta}}<1 .
$$

Besides, by (69), we know, for $0<\delta<\frac{1}{2}$, that $u_{0}^{\delta} \rightarrow 0$ uniformly as $r=|x| \rightarrow \infty$. Thus $e^{\xi\left(u_{0}^{\delta}\right) u_{0}^{\delta}} \rightarrow 1$ uniformly as $r \rightarrow \infty$. Hence

$$
\begin{aligned}
e^{-a w_{0}-a e^{u_{0}^{\delta}}}\left(e^{u_{0}^{\delta}}-1\right) \leqq & e^{-a w_{0}-a}\left(e^{u_{0}^{\delta}}-1\right) \\
= & e^{-a w_{0}-a+\xi\left(u_{0}^{\delta}\right) u_{0}^{\delta}} u_{0}^{\delta} \\
= & e^{-a+\xi\left(u_{0}^{\delta}\right) u_{0}^{\delta}} u_{0}^{\delta}\left(\prod_{l=1}^{m} \frac{1}{\left(1+\left|x-p_{l}\right|^{2}\right)^{a n_{l}}}\right) \\
(\text { by }(69))= & -e^{-a+\xi\left(u_{0}^{\delta}\right) u_{0}^{\delta}}\left(\sum_{l=1}^{m} \frac{n_{l}}{1+\xi_{l}} \cdot \frac{1-\delta}{\delta+\left|x-p_{l}\right|^{2}}\right) \\
& \times\left(\prod_{l=1}^{m} \frac{1}{\left(1+\left|x-p_{l}\right|^{2}\right)^{a n_{l}}}\right) \\
\equiv & -h_{\delta},
\end{aligned}
$$

where

$$
\xi_{l}=\theta_{l}\left(\frac{1-\delta}{\delta+\left|x-p_{l}\right|^{2}}\right) \quad \text { with } 0<\theta_{l}<1, \quad l=1, \ldots, m .
$$

By virtue of the assumption $a N \leqq 1$ or $2 a N+2 \leqq 4$, we obtain

$$
\left.r^{4} h_{\delta}(x) \rightarrow \infty \quad \text { (if } a N<1\right) \text { as } r=|x| \rightarrow \infty
$$

uniformly with respect to $0<\delta<1 / 2$ or

$$
r^{4} h_{\delta}(x) \rightarrow \text { some number } c_{\delta}>0 \quad \text { (if } a N=1 \text { ) as } r=|x| \rightarrow \infty .
$$

Using the definition of $h_{\delta}$, it is easily seen that there is a suitable $c_{0}$ so that $c_{\delta} \geqq c_{0}$ for all $0<\delta<\frac{1}{2}$.

The above observation enables us to conclude that there is an $r_{0}>0$ and $\lambda_{1}>0$ so that

$$
\lambda e^{-a w_{0}-a e^{u_{0}^{\delta}}}\left(e^{u_{0}^{\delta}}-1\right)+g \leqq-\frac{1}{r^{4}}\left(\lambda r^{4} h_{\delta}-r^{4} g\right)<0
$$

whenever $r=|x| \geqq r_{0}$ and $\lambda>\lambda_{1}$ because $r^{4} g=\mathrm{O}(1)$ at infinity.

On the other hand, we see by the definition of $u_{0}^{\delta}$ that $u_{0}^{\delta} \leqq u_{0}^{\frac{1}{2}}\left(\delta \leqq \frac{1}{2}\right)$. Thus

$$
e^{-a w_{0}-a e^{u_{0}^{\delta}}}\left(e^{u_{0}^{\delta}}-1\right) \leqq e^{-a w_{0}-a}\left(e^{u_{0}^{\frac{1}{2}}}-1\right) .
$$

By (71), we can find $\lambda_{0} \geqq \lambda_{1}$ so that (68) holds in $\left\{x \in \mathbf{R}^{2}|| x \mid<r_{0}\right\}$ for all $0<\delta<1 / 2$ whenever $\lambda>\lambda_{0}$. 
The lemma is proven.

5.3. Solution of the Original Equation - the Nonradial Case. Since $v_{1}^{\delta}>0$ is a supersolution, $v=0$ is a subsolution, and all involved are regular, so by [Ni2], we see that (66) has a smooth solution $v^{\delta}$ in $\mathbf{R}^{2}$ satisfying

$$
-u_{0}^{\delta}=v_{1}^{\delta} \geqq v^{\delta} \geqq 0 \quad \text { in } \mathbf{R}^{2} .
$$

We now study the passage $\delta \rightarrow 0$ of the family $\left\{v^{\delta}\right\}$.

For $\delta_{1}<\delta_{2}$, we have $u_{0}^{\delta_{1}}<u_{0}^{\delta_{2}}$. Hence $v_{1}^{\delta_{1}}>v_{1}^{\delta_{2}}$. In particular, $v_{0} \equiv-u_{0}=$ $v_{1}^{0}>v_{1}^{\delta}$ for all $\delta>0$. Thus a weaker form of (72) is

$$
v_{0}>v^{\delta} \geqq 0 \quad \text { in } \mathbf{R}^{2}
$$

Let us consider the right-hand side of (66). It is clear that

$$
e^{-a e^{u_{0}^{\delta}+v^{\delta}}}\left(e^{u_{0}^{\delta}+v^{\delta}}-1\right)
$$

is a bounded function with an upper bound independent of $\delta$. Besides, we have in view of (73) the bound

$$
\begin{aligned}
e^{-a w_{0}+a v^{\delta}} & \leqq e^{-a w_{0}+a v_{0}}=e^{-a\left(u_{0}+w_{0}\right)} \\
& =\prod_{l=1}^{m}\left|x-p_{l}\right|^{-2 a n_{l}}=f
\end{aligned}
$$

for the other factor on the right-hand side of (66). So the $\delta$-independent upper bound function $f$ has singularities at $x=p_{l}, l=1, \ldots, m$. We hope to apply $L^{p}$-estimates to control the sequence $\left\{v^{\delta}\right\}$. For this purpose, we require

$$
f \in L_{\mathrm{loc}}^{p}\left(\mathbf{R}^{2}\right) \quad \text { for some } p>1 \text {. }
$$

By (74) and $n_{l} \leqq N$, we see that (75) is ensured provided that $a N<1$. When $a N=1$, we only consider the nonradial case where $m \geqq 2$ (there are more than two centers of strings). Thus $n_{l}<N$ for $l=1, \ldots, m$, and (75) is still ensured. Roughly speaking, the condition $a N \leqq 1$ is sufficient to give us (75). It is interesting to note that this local regularity condition is the same as the condition stated in Lemma 5.2 where we need to control the behavior of the nonlinearity at infinity in order to obtain a subsolution.

As a consequence, we conclude that the right-hand side of (66) has uniform $L^{p}$ bound on any given compact domain in $\mathbf{R}^{2}$. In other words, for any bounded domain $\mathcal{O} \subset \mathbf{R}^{2}$, there is a constant $C(p, \mathcal{O})>0$ independent of $\delta$, so that

$$
\left\|\Delta v^{\delta}\right\|_{L^{p}(\mathbb{C})} \leqq C(p, \mathcal{O})
$$

Applying the interior $L^{p}$-estimates $[\mathrm{ADN}, \mathrm{BJS}]$ and using (73) and (76), we see that

$$
\left\|v^{\delta}\right\|_{W^{2, p(\mathcal{C})}} \leqq C(p, \mathcal{O})
$$


for some $\delta$-independent constant $C(p, \mathcal{O})>0$. Using the continuous embedding

$$
W^{k, p}(\mathcal{O}) \rightarrow C^{m}(\overline{\mathcal{O}}) \text { for } 0 \leqq m<k-\frac{2}{p}
$$

with $k=2$, we see that $\left\{v^{\delta}\right\}$ is bounded in $C(\overline{\mathcal{O}})$. From this fact and (73), we conclude that $\left\{v^{\delta}\right\}$ is uniformly bounded over the full $\mathbf{R}^{2}$.

In view of $(66)$, the boundedness of $\left\{v^{\delta}\right\}$ implies that $\left\{\Delta v^{\delta}\right\}$ is also bounded in $\mathbf{R}^{2}$. Thus the interior $L^{p}$-estimates say that (77) holds for any $p>1$ and any given bounded domain $\mathcal{O}$. Take $p>2$. The embedding (78) gives us the bound

$$
\left|v^{\delta}\right|_{C^{1}(\bar{C})} \leqq C(p, \mathcal{O}), \quad \forall \delta>0 .
$$

Using the properties of the right-hand side of (66), we see in view of (79) that $\left|\Delta v^{\delta}\right|_{C^{1}(\overline{\mathcal{C}})} \leqq C(p, \mathcal{O})$ for any $\delta$. Since $\mathcal{O}$ is arbitrary, the above results and the interior Schauder estimates enable us to conclude that for each $\alpha \in(0,1)$ there is a constant $C(\alpha, p, \mathcal{O})$ independent of $\delta>0$ so that

$$
\left|v^{\delta}\right|_{C^{2, \alpha}(\overline{\mathcal{C}})} \leqq C(\alpha, p, \mathcal{O}) .
$$

We are now ready to use a standard diagonal subsequence argument to obtain a solution of $(65)$ on $\mathbf{R}^{2}$ in the limit $\delta \rightarrow 0$.

Let $r_{1}<r_{2}<\cdots<r_{i}<\cdots, r_{i} \rightarrow \infty$ (as $\left.i \rightarrow \infty\right)$ be a sequence of positive numbers and

$$
B_{i}=\left\{x \in \mathbf{R}^{2}|| x \mid<r_{i}\right\} .
$$

Applying the estimate $(80)$ on $\left\{v^{\delta}\right\}$ with $\mathcal{O}=B_{l}, i=1,2, \ldots$, and the compact embedding $C^{2, \alpha}\left(\bar{B}_{i}\right) \rightarrow C^{2}\left(\bar{B}_{i}\right)$, we can extract on each $B_{l}$ a convergent subsequence of $\left\{v^{\delta}\right\}$ in $C^{2}\left(\bar{B}_{l}\right)$. We start from $B_{1}$. Choose $\delta_{n}^{1}, \delta_{n}^{1} \rightarrow 0$ as $n \rightarrow \infty$ and $v_{1} \in C^{2}\left(\overline{B_{1}}\right)$ so that $v^{\delta_{n}^{1}} \rightarrow v_{1}$ in $C^{2}\left(\overline{B_{1}}\right)$ as $n \rightarrow \infty$. Then there is a subsequence $\left\{\delta_{n}^{2}\right\}$ (say) of $\left\{\delta_{n}^{1}\right\}$ and an element $v_{2} \in C^{2}\left(\overline{B_{2}}\right)$ satisfying $\delta_{n}^{2} \rightarrow \infty$ and $v^{\delta_{n}^{2}} \rightarrow v_{2}$ in $C^{2}\left(\overline{B_{2}}\right)$ as $n \rightarrow \infty$. Of course, $v_{1}=v_{2}$ in $B_{1}$. We can repeat this procedure to get sequences $\left\{\delta_{n}^{l}\right\}, i=1,2, \ldots$, so that

(i) $\left\{\delta_{n}^{i}\right\} \subset\left\{\delta_{n}^{i-1}\right\}, i=2,3, \ldots$;

(ii) for each fixed $i=1,2, \ldots, \delta_{n}^{i} \rightarrow 0$ as $n \rightarrow \infty$;

(iii) for each fixed $i=1,2, \ldots$, there is an element $v_{l} \in C^{2}\left(\widehat{B_{l}}\right)$ satisfying $v^{\delta_{n}^{l}} \rightarrow$ $v_{i}$ as $n \rightarrow \infty$;

(iv) there holds $v_{l}=v_{i-1}$ on $B_{i-1}, i=2,3, \ldots$.

Set $v(x)=v_{i}(x)$ for $x \in B_{i}$ and $i=1,2, \ldots$. The property (iv) above says that $v$ is a well-defined $C^{2}$-function on $\mathbf{R}^{2}$. By virtue of (i) and (iii), we see that $v^{\delta_{n}^{n}} \rightarrow v$ as $n \rightarrow \infty$ in $C^{2}(\overline{\mathcal{O}})$-norm for any given bounded domain $\mathcal{O}$ in $\mathbf{R}^{2}$. Take $\delta=\delta_{n}^{n}$ in (66). Letting $n \rightarrow \infty$ and using (i) above, we find that $v$ is a smooth solution of (65). Moreover, the inequality (73) implies that $v$ verifies the same bounds

$$
0 \leqq v \leqq-u_{0} \quad \text { in } \mathbf{R}^{2}
$$

In particular, $v$ vanishes at infinity. 
5.4. Solution of the Radial Case. We now deal with the case $a N=1$ and $m=1$ (clustered strings) individually. Without loss of generality, we assume that the single center of the $N$ strings is at the origin. It suffices to find a radially symmetric solution. For this purpose, we use the reduction in [CHMcY] with $u_{0}=2 N \ln r(r=|x|)$. Then Eqs. (62)-(63) give us

$$
\frac{1}{a} \eta+e^{u}-u+2 N \ln r=c=\text { constant }
$$

Thus, we come up with a single equation, replacing (65) with $\lambda=e^{a c}$ as before,

$$
\begin{aligned}
& u_{r r}+\frac{1}{r} u_{r}=\lambda r^{-2 a N} e^{a\left(u-e^{u}\right)}\left(e^{u}-1\right), \quad r>0, \\
& \lim _{r \rightarrow 0} r u_{r}(r)=2 N, \quad \lim _{r \rightarrow \infty} u(r)=0 .
\end{aligned}
$$

The boundary condition at $r=0$ is important when we use the radial solution defined in the punctured plane $r>0$ to get a classical solution of the original problem over the entire $\mathbf{R}^{2}$. For a full discussion of this simple "removable singularity" property, see $[\mathrm{SY}]$ for a similar problem.

We now introduce the new variables

$$
t=\ln r, \quad U(t)=u\left(e^{t}\right) .
$$

Then the system (82) becomes

$$
\begin{aligned}
& U^{\prime \prime}=\lambda e^{a\left(U-e^{U}\right)}\left(e^{U}-1\right), \quad-\infty<t<\infty, \\
& \lim _{t \rightarrow-\infty} U^{\prime}(t)=2 N, \quad \lim _{t \rightarrow \infty} U(t)=0 .
\end{aligned}
$$

In [CHMcY], we showed that Eq. (83) has a negative solution in the neighborhood of $t=-\infty$ and $\lim _{t \rightarrow-\infty} U(t)=-\infty$. Besides, note that the right-hand side of the equation, $g(U)$, can be written in the form

$$
g(U)=\lambda e^{a\left(U-e^{U}\right)}\left(e^{U}-1\right)=-\frac{\lambda}{a} \frac{\mathrm{d}}{\mathrm{d} U}\left[e^{a\left(U-e^{U}\right)}\right] .
$$

Therefore, multiplying Eq. (83) by $U^{\prime}$ and integrating over $(-\infty, t)$, we find the reduced equation

$$
U^{\prime 2}(t)=4 N^{2}-\frac{2 \lambda}{a} e^{a\left(U-e^{U}\right)} \equiv F(U) .
$$

It will be useful to study the critical points of this equation first. Suppose $\bar{U}$ is a number that $F(\bar{U})=0$. In order to ensure the uniqueness property at the equilibrium $U=\bar{U}$ for (84), we need to require that

$$
F^{\prime}(\bar{U})=2 \lambda\left(e^{\bar{U}}-1\right) e^{a\left(\bar{U}-e^{\bar{U}}\right)}=0 .
$$

The only choice is $\bar{U}=0$. Inserting this result into $F(\bar{U})=0$, we find that

$$
\lambda=2 a N^{2} e^{a}=2 N e^{\frac{1}{N}},
$$


where we have used the condition $a N=1$. In the sequel, we will always assume (85). Hence, for $t$ in a neighborhood of $t=-\infty$, we can rewrite (84) in the following form so that the derivative is explicit:

$$
U^{\prime}(t)=2 N \sqrt{1-e^{\frac{1}{N}\left(1+U-e^{U}\right)}}
$$

where we have chosen the positive radical root because, according to the boundary condition at $t=-\infty$ in (83), $U^{\prime}>0$ initially. Since, in (86), $U^{\prime}>0$ and the uniqueness holds at the equilibrium $\bar{U}=0$, we can use the fact that $F(U)$ decreases in $U<0$ to conclude that $U=U(t)$ (the local solution of (82) near $t=-\infty$ ) solves (86) in the entire interval $-\infty<t<\infty$ and $U(t)<0=\bar{U}$ for all $t$.

Besides, (86) can be rewritten in the integral form

$$
\int_{U(0)}^{U(t)} \frac{\mathrm{d} U}{\sqrt{F(U)}}=t .
$$

Consequently, we must have $U(t) \rightarrow \bar{U}=0$ as $t \rightarrow \infty$. In fact, since

$$
F^{\prime \prime}(0)=2 \lambda e^{-a}=4 N
$$

we can derive from (87) the sharp estimate

$$
|U|=\mathrm{O}\left(e^{-\sqrt{2 N} t}\right) \quad \text { as } t \rightarrow \infty .
$$

Returning to the original variable $r=e^{t}, u(r)=U(\ln r)$, it is seen that a desired solution of (62)-(63) is obtained. However, we do not have a bound like (81). Instead, from (88), we have

$$
|u(r)|=\mathrm{O}\left(r^{-\sqrt{2 N}}\right) \quad \text { as } r \rightarrow \infty
$$

Note also that (86) implies $U^{\prime}(t) /|U(t)| \rightarrow \sqrt{F^{\prime \prime}(0) / 2}=\sqrt{2 N}$ as $t \rightarrow \infty$. Hence

$$
\left|u_{r}(r)\right|=\mathrm{O}\left(r^{-(1+\sqrt{2 N})}\right) \quad \text { as } r \rightarrow \infty .
$$

In order to see that the solutions just obtained carry finite energies, we need to study the asymptotic behavior of $v$ in (81) or $u$ itself.

\section{Proof of Theorem 2.3: Asymptotic Decay Estimates}

In this section, we complete the proof of Theorem 2.3 by obtaining the asymptotic properties of the solutions and the condition that is crucial for the gravitational metric to be complete. We split out discussion into the following two subsections.

6.1. Decay Estimates. Let $v$ be a solution of (65) satisfying (81). Then $u=u_{0}+v$ fulfills Eq. (62) with the function $\eta$ given by the expression

$$
\eta=-a w_{0}+a\left(v-e^{u_{0}+v}\right)+c
$$


Hence, by the definition of $w_{0}$, we have

$$
e^{\eta}=e^{c+a\left(v-e^{u_{0}+v}\right)} \prod_{l=1}^{m} \frac{1}{\left(1+\left|x-p_{l}\right|^{2}\right)^{a n_{l}}} .
$$

Choose $r_{0}>0$ sufficiently large so that

$$
\left\{p_{1}, \ldots, p_{m}\right\} \subset B\left(r_{0}\right)=\left\{x \in \mathbf{R}^{2}|| x \mid<r_{0}\right\} .
$$

Then (62) becomes

$$
\Delta u=e^{\eta}\left(e^{u}-1\right) \quad \text { in } \mathbf{R}^{2}-\overline{B\left(r_{0}\right)} .
$$

The decay property (92) implies the following.

Lemma 6.1. Suppose that $a N<1$. Then the solution $u$ of (93) has the bounds

$$
-C_{b}|x|^{-b}<u(x)<0, \quad|x|>r_{0}
$$

for any $b>0$. Here $C_{b}>0$ is a constant depending on $b$. If $a N=1$ and $m \geqq 2$ (there are at least two string centers), (94) holds for $b=2$. If $a N=1$ but $m=1$ (superimposed strings), then the radial solution satisfies (94) with $b=\sqrt{2 N}$.

Proof. Assume $a N<1$ first. Introduce the comparison function

$$
w(x)=C|x|^{-b} .
$$

Then

$$
\Delta w=b^{2} r^{-2} w, \quad|x|=r>r_{0}
$$

Choose $\xi \in[0,1]$ so that $e^{u}-1=e^{\xi u} u$. Thus, by (93), (96), and setting $\sigma=e^{\eta+\xi u}$, we have for $r_{0}>0$ sufficiently large,

$$
\begin{aligned}
\Delta(u+w) & =\sigma u+b^{2} r^{-2} w \\
& <b^{2} r^{-2}(u+w), \quad|x|=r>r_{0},
\end{aligned}
$$

since $e^{\eta}$ satisfies $(92)$ and $2 a N<2$. For such fixed $r_{0}$, we can take the constant $C$ in (95) large to make

$$
\left.(u(x)+w(x))\right|_{|x|=r_{0}}>0 .
$$

Applying the maximum principle in (97), we obtain $u+w>0$ in $\mathbf{R}^{2}-\overline{B\left(r_{0}\right)}$ as expected.

If $a N=1$ but $m \geqq 2$, the estimate comes from (81) and the definition of $u_{0}$ (see the proof of Lemma 6.2 below). If $a N=1$ and $m=1$, the estimate follows from (89).

We next show that $\partial_{j} u$ satisfies similar decay properties as $u$ stated in (94). For this purpose, we first prove

Lemma 6.2. Suppose $a N<1$ or aN=1 but $m \geqq 2$. There holds $\partial_{j} v=0$ at infinity, $j=1,2$.

Proof. By (81) and

$$
-u_{0}(x)=\sum_{l=1}^{m} n_{l} \ln \left(1+\frac{1}{\left|x-p_{l}\right|^{2}}\right)=\mathrm{O}\left(r^{-2}\right)
$$


as $|x|=r \rightarrow \infty$, we see that $v \in L^{2}\left(\mathbf{R}^{2}\right)$. Besides, using

$$
\begin{aligned}
0 \leqq 1-e^{u_{0}+v} & \leqq 1-e^{u_{0}} \\
& =1-\prod_{l=1}^{m} \frac{\left|x-p_{l}\right|^{2 n_{l}}}{\left(1+\left|x-p_{l}\right|^{2}\right)^{n_{l}}}=\mathrm{O}\left(r^{-2}\right)
\end{aligned}
$$

and

$$
e^{-a w_{0}}=\prod_{l=1}^{m} \frac{1}{\left(1+\left|x-p_{l}\right|^{2}\right)^{a n_{l}}}=\mathrm{O}\left(r^{-2 a N}\right)
$$

(at $|x|=r=\infty$ ), we conclude that the right-hand side of (65) lies in $L^{2}\left(\mathbf{R}^{2}\right)$ as well. Hence the $L^{2}$-estimates for (65) enable us to get $v \in W^{2,2}\left(\mathbf{R}^{2}\right)$.

Furthermore, differentiating (65) gives us

$$
\begin{aligned}
\Delta\left(\partial_{J} v\right)= & \lambda e^{-a w_{0}+a\left(v-e^{u_{0}+v}\right)}\left(e^{u_{0}+v}-a\left[e^{u_{0}+v}-1\right]^{2}\right)\left(\partial_{j} v\right) \\
& +\lambda e^{-a w_{0}+a\left(v-e^{u_{0}+v}\right.}\left(1+a-a e^{u_{0}+v}\right) e^{v}\left(\partial_{j} e^{u_{0}}\right) \\
& +\lambda e^{a\left(v-e^{u_{0}+v}\right)}\left(e^{u_{0}+v}-1\right)\left(\partial_{j} e^{-a w_{0}}\right)+\left(\partial_{j} g\right) .
\end{aligned}
$$

Of course, $\partial_{,} g \in L^{2}\left(\mathbf{R}^{2}\right)$. Using

$$
\partial_{j} e^{u_{0}}=\partial_{j}\left(\prod_{l=1}^{m} \frac{\left|x-p_{l}\right|^{2 n_{l}}}{\left(1+\left|x-p_{l}\right|^{2}\right)^{n_{l}}}\right)=\mathrm{O}\left(r^{-1}\right)
$$

and (98), we find

$$
e^{-a w_{0}}\left(\partial_{j} e^{u_{0}}\right)=\mathrm{O}\left(r^{-(2 a N+1)}\right) \in L^{2}\left(\mathbf{R}^{2}\right) .
$$

Differentiating (98) gives us

$$
\partial_{j} e^{-a w_{0}}=\mathrm{O}\left(r^{-(2 a N+1)}\right) .
$$

Inserting the above information into (99), we see that the right-hand side of (99) lies in $L^{2}\left(\mathbf{R}^{2}\right)$. Thus the elliptic $L^{2}$-estimates lead us to the conclusion $\partial_{j} v \in W^{2,2}\left(\mathbf{R}^{2}\right)$. Consequently $\partial_{j} v \rightarrow 0$ as $|x| \rightarrow \infty$.

We are now ready to derive the decay estimates for $|\nabla u|$.

Lemma 6.3. For the solution $u$ of (93), we have

$$
|\nabla u|^{2} \leqq C_{b}|x|^{-b}, \quad|x|>r_{0},
$$

where $b>0$ is again arbitrary for $a N<1$ and $C_{b}>0$ is a constant, while, for $a N=1$ but $m \geqq 2$, the solution of (93) can be so obtained that (100) holds for $b=3$.

Proof. From Lemma 6.2 and the definition of $u_{0}$, we see that $|\nabla u| \rightarrow 0$ as $|x| \rightarrow \infty$. Differentiating (93), we obtain

$$
\begin{aligned}
\Delta\left(\partial_{j} u\right) & =\left(\partial_{j} \eta\right) e^{\eta}\left(e^{u}-1\right)+e^{\eta+u}\left(\partial_{j} u\right) \\
& =f_{j}+\left(e^{\eta+u}-a e^{\eta}\left(e^{u}-1\right)^{2}\right)\left(\partial_{j} u\right) .
\end{aligned}
$$


In view of (91) and Lemma 6.1, we see that, at infinity,

$$
f_{J}= \begin{cases}\mathrm{O}\left(r^{-b_{1}}\right), & \forall b_{1}>0, \text { when } a N<1, \\ \mathrm{O}\left(r^{-5}\right), & \text { when } a N=1, m \geqq 2 .\end{cases}
$$

Set $h=|\nabla u|^{2}$. Thus, as a consequence of (101), there holds

$$
\begin{aligned}
\Delta h & \geqq 2 e^{\eta+u} h+2(\nabla u \cdot \nabla \eta) e^{\eta}\left(e^{u}-1\right) \\
& \geqq e^{\eta+u} h+q(x), \quad|x|>r_{0}
\end{aligned}
$$

where $q(x)$ satisfies the same decay estimate as $f_{j}$ in (102).

Suppose that $w$ is given by (95). From (96) and (103), we have

$$
\Delta(h-w) \geqq e^{\eta+u} h-b^{2} r^{-2} w+q(x), \quad|x|=r>r_{0} .
$$

Assume that $b_{1}>2+b$ when $a N<1$ or $5 \geqq 2+b$ when $a N=1$ but $m \geqq 2$. Then there is some $C>0$ in (95) so that

$$
q(x)>-b^{2} r^{-2} w(x) \text { for }|x|=r>r_{0} .
$$

By (92), if $a N<1$, we may also assume that

$$
e^{\eta+u}>2 b^{2} r^{-2} \text { for }|x|=r>r_{0}
$$

while, if $a N=1$ but $m \geqq 2$, by the uniform bound (81) and the arbitrariness of the constant $c$ in (91) or (92) so that $\left.\left(e^{\eta(x)}\right)\right|_{|x|=r_{0}}$ may be made sufficiently large, we still have the validity of (106).

Inserting (105)-(106) into (104), we find the inequality

$$
\begin{aligned}
\Delta(h-w) & \geqq e^{\eta+u} h-2 b^{2} r^{-2} w \\
& \geqq 2 b^{2} r^{-2}(h-w), \quad|x|=r>r_{0} .
\end{aligned}
$$

Of course, we can adjust the constant $C$ in (95) to make

$$
\left.(h(x)-w(x))\right|_{|x|=r_{0}} \leqq 0 .
$$

Using the boundary conditions (108) and $h-w \rightarrow 0$ (as $|x| \rightarrow \infty$ ) in (107), we arrive at $h \leqq w$ for $|x|>r_{0}$ as desired.

Let $u=u_{0}+v$ and $\eta$ be defined in (91). Then $g_{j k}=e^{\eta} \delta_{j k}$ and $(\phi, A)$ give rise to a solution triplet of the coupled Einstein and Abelian Higgs equations (1) or (5) on the conformally flat surface $\left(\mathbf{R}^{2},\left\{g_{J k}\right\}\right)$, where

$$
\begin{aligned}
\phi(x) & =\exp \left(\frac{1}{2} u(x)+\mathrm{i} \sum_{l=1}^{m} n_{l} \arg \left(x-p_{l}\right)\right), \\
A_{1}(x) & =-\operatorname{Re}\left\{2 \mathrm{i} \partial^{*} \ln \phi\right\}, \quad A_{2}(x)=-\operatorname{Im}\left\{2 \mathrm{i} \partial^{*} \ln \phi\right\} .
\end{aligned}
$$


In general, if $\phi$ has the local representation $\phi=e^{\sigma+\mathrm{i} \omega}$ where $\sigma$ and $\omega$ are real functions, by $\partial_{1}=\partial+\partial^{*}, \partial_{2}=\mathrm{i}\left(\partial-\partial^{*}\right)$ and the second line in (109), we have

$$
\begin{aligned}
& D_{1} \phi=\left(\partial+\partial^{*}\right) \phi-\mathrm{i}\left\{-\mathrm{i} \frac{\partial^{*} \phi}{\phi}+\mathrm{i} \frac{\partial \phi^{*}}{\phi^{*}}\right\} \phi=2 \phi \partial \sigma, \\
& D_{2} \phi=\mathrm{i}\left(\partial-\partial^{*}\right) \phi-\mathrm{i}\left\{-\mathrm{i} \frac{\partial^{*} \phi}{\phi}-\frac{\partial \phi^{*}}{\phi^{*}}\right\} \phi=2 \mathrm{i} \phi \partial \sigma .
\end{aligned}
$$

Using these formulas and (109), we have the relation $\left|D_{1} \phi\right|^{2}+\left|D_{2} \phi\right|^{2}=\frac{1}{2} e^{u}|\nabla u|^{2}$.

Let $b$ be the exponent described in Lemma 6.1 . Then by the third equation in (24) and (92), we have

$$
\begin{aligned}
F_{12} & =\mathrm{O}\left(r^{(-b+2 a N)}\right), \\
1-|\phi|^{2} & =\mathrm{O}\left(r^{-b}\right)
\end{aligned}
$$

for $r=|x|$ large. By Lemma 6.3, we have

$$
\left|D_{j} \phi\right|^{2}= \begin{cases}\mathrm{O}\left(r^{-b}\right), & \forall b>0, \quad \text { where } a N<1, \\ \mathrm{O}\left(r^{-3}\right), & \text { where } a N=1, m \geqq 2, \\ \mathrm{O}\left(r^{-2(1+\sqrt{2 N})}\right), & \text { where } a N=1, m=1\end{cases}
$$

These estimates imply immediately that

$$
\int_{\mathbf{R}^{2}} F_{12} \mathrm{~d} x=2 \pi N
$$

Consequently, by the first equation in (24) and (92), we find that the Gauss curvature satisfies

$$
K_{g}= \begin{cases}\mathrm{O}\left(r^{-b}\right), & \forall b>0, \quad \text { where } a N<1, \\ \mathrm{O}\left(r^{-1}\right), & \text { where } a N=1, m \geqq 2, \\ \mathrm{O}\left(r^{-2 \sqrt{2 N}}\right), & \text { where } a N=1, m=1 .\end{cases}
$$

Thus the decay estimates stated in Theorem 2.3 are obtained.

Furthermore, since we can rewrite the energy density of the matter-gauge sector in the form

$$
\begin{aligned}
\mathscr{E}= & \frac{1}{2} e^{-2 \eta} F_{12}^{2}+\frac{1}{2} e^{-\eta}\left(\left|D_{1} \phi\right|^{2}+\left|D_{2} \phi\right|^{2}\right)+\frac{1}{8}\left(|\phi|^{2}-1\right)^{2} \\
= & \frac{1}{2}\left(e^{-\eta} F_{12}+\frac{1}{2}\left(|\phi|^{2}-1\right)\right)^{2}+\frac{1}{2} e^{-\eta}\left|D_{1} \phi+\mathrm{i} D_{2} \phi\right|^{2} \\
& -\frac{1}{2} e^{-\eta} F_{12}\left(|\phi|^{2}-1\right)+\frac{\mathrm{i}}{2} e^{-\eta}\left(\left[D_{1} \phi\right]\left[D_{2} \phi\right]^{*}-\left[D_{1} \phi\right]^{*}\left[D_{2} \phi\right]\right) \\
= & \frac{1}{2} e^{-\eta} F_{12}+\frac{1}{2} e^{-\eta} \operatorname{Im}\left\{\partial_{J} \varepsilon^{j k} \phi^{*}\left(D_{k} \phi\right)\right\},
\end{aligned}
$$

hence (110)-(111) lead us to the following total energy of the matter-gauge coupling

$$
\int_{\mathbf{R}^{2}} \mathscr{E} e^{\eta} \mathrm{d} x=\pi N
$$

and the energy of the gravitational sector which is realized by the total Gauss 
curvature

$$
\int_{\mathbf{R}^{2}} K_{g} e^{\eta} \mathrm{d} x=4 \pi^{2} G N
$$

where we have used the first equation in (24) to relate the total curvature to the matter-gauge energy. Returning to the original system with the symmetry-breaking parameter $\varepsilon>0$, we see that the decay estimates and the quantization identities are established.

6.2. The Completeness of the Metric. Let $p, q$ be two arbitrarily given points in the conformally flat surface $\left(\mathbf{R}^{2}, e^{\eta} \delta_{j k}\right)$, where the conformal exponent $\eta$ is determined by the expression (91). Suppose that $\mathscr{C}$ is the set of piecewise differentiable curves of the form $x=x(t) \in \mathbf{R}^{2}, 0 \leqq t \leqq T$ connecting $p, q$. Namely, $x(0)=p, x(T)=q$. The geodesic metric $d$ on $\left(\mathbf{R}^{2}, e^{\eta} \delta_{j k}\right)$ is defined by

$$
d(p, q)=\inf _{x \in \mathscr{C}} \int_{0}^{T} e^{\frac{1}{2} \eta(x(t))}|\dot{x}(t)| \mathrm{d} t, \quad p, q \in \mathbf{R}^{2} .
$$

Recall that $\left(\mathbf{R}^{2}, e^{\eta} \delta_{j k}\right)$ is called complete if it is a complete metric space with respect to the metric $d$ and, by the Hopf-Rinow-de Rham theorem, this latter property is equivalent to the statement that each geodesic on $\left(\mathbf{R}^{2}, e^{\eta} \delta_{j k}\right)$ can be extended to a global geodesic defined on the entire real line R. See e.g. [S]. In this case, the infimum in (112) may always be attained by minimizing geodesics, which is obviously an important feature.

We now proceed to show that the metric completeness for the solutions obtained is equivalent to the restriction $N \leqq 1 / 2 \pi \varepsilon^{2} G$. in $\mathbf{R}^{2}$ :

In fact, for the obtained solutions, the conformal factor $e^{\eta}$ satisfies the estimates

$$
C_{1}(1+r)^{-2 a N} \leqq e^{\eta(x)} \leqq C_{2}(1+r)^{-2 a N}, \quad r=|x|,
$$

where $C_{1}, C_{2}>0$ are constants. By (113), it suffices to consider the radially symmetric metric $g_{0}=(1+r)^{-2 a N} \delta_{j k}$.

Considering rays starting from the origin as geodesics, it is straightforward to see that the completeness of $g_{0}$ is equivalent to the property that the geodesic distance from the origin to infinity is infinite, namely, the integral

$$
\int_{0}^{\infty}(1+r)^{-a N} \mathrm{~d} r
$$

is divergent, which clearly requires that $a N \leqq 1$ as expected.

Thus we see that the obtained gravitational metric is complete if and only if the total string or vortex number $N$ satisfies the upper bound $N \leqq 1 / 2 \pi \varepsilon^{2} G$, which coincides with the existence condition stated in Theorem 2.3.

The proof of Theorem 2.3 is complete.

Remarks. (a) The Gauss curvature $K_{g}$ obtained in Theorem 2.3 decays fast at infinity. Such a property already implies that $K_{g}$ is the curvature of a complete metric $g^{\prime}$ (say) because a well-known theorem [KW2] says that $K_{g} \in C^{\infty}\left(\mathbf{R}^{2}\right)$ is the curvature of a complete Riemannian metric on $\mathbf{R}^{2}$ if and only if

$$
\lim _{r \rightarrow \infty} \inf _{|x| \geqq r} K_{g}(x) \leqq 0
$$


In our case considered here, we can have $g^{\prime}=g$ when $N \leqq 1 / 2 \pi \varepsilon^{2} G$. On the other hand, when $K_{g}$ decays fast at infinity but $N \leqq 1 / 2 \pi \varepsilon^{2} G$ is violated, the gravitational metric $g$ will fail to be complete. Thus, now, $g \neq$ the complete metric $g^{\prime}$ mentioned above.

(b) In an earlier paper [CHMcY], we have constructed all finite-energy radially symmetric $N$-string solutions in the ranges $0<N<1 / 2 \pi \varepsilon^{2} G$ and $1 / 2 \pi \varepsilon^{2} G<N<$ $1 / \pi \varepsilon^{2} G$. There the conformal exponent $\eta$ of the gravitational metric is expressed in the form.

$$
\eta=-2 a N \ln r+a\left(u-e^{u}\right)+c, \quad a=2 \pi \varepsilon^{2} G, \quad r=|x|, x \in \mathbf{R}^{2}
$$

so that the function $u$ satisfies the property $u=2 N \ln r+\mathrm{O}(1)$ at $r=0$ and $\eta$ is everywhere regular. In fact in [CHMcY] the constant $c$, which clearly defines a metric "background," is set to zero for convenience. This point of view comes from the assumption that one should recover the Minkowski metric represented by $\eta=0$ when gravity is turned off through putting $G=0$. In this way it is shown that there is energy blow-up at $N=1 / a=1 / 2 \pi \varepsilon^{2} G$ too. In the present paper we have proven that when $c$ is suitably chosen, there are finite-energy solutions in the full range $N \leqq 1 / a=1 / 2 \pi \varepsilon^{2} G$. It is straightforward to examine that the conclusions arrived in [CHMcY] in the ranges $0<N<1 / a=1 / 2 \pi \varepsilon^{2} G$ and $1 / 2 \pi \varepsilon^{2} G<N<1 / \pi \varepsilon^{2} G$ as well as in $N \geqq 1 / \pi \varepsilon^{2} G$ are independent of the value of $c$. Thus we are led to the obstruction result stated in Theorem 2.1. The equation $N=1 / 2 \pi \varepsilon^{2}$, however, may be read as an obstruction to an arbitrary choice of the metric background. This is a peculiar and unique situation for radially symmetric solutions.

When $a N<1$, the solutions with $u \rightarrow 0$ as $r \rightarrow \infty$ correspond to the solutions of the coupled Einstein and Abelian Higgs equations (1) for which the Higgs field go to the asymmetric vacuum. Such solutions are contained in those already discussed in the present paper. The other family of solutions are those which have the asymptotic behavior

$$
\lim _{r \rightarrow \infty} r u_{r}=-\beta, \quad \beta>\frac{2(2-a N)}{a} .
$$

Thus, by (114)-(115), we obtain the sharp decay estimate for the conformal factor $e^{\eta}$ as follows:

$$
e^{\eta}=\mathrm{O}\left(r^{-\alpha}\right) \text { as } r \rightarrow \infty, \quad \text { where } \alpha>2 a N+2(2-a N)=4 .
$$

Combining the above with the discussion in this section, we see that $\left(\mathbf{R}^{2}, e^{\eta} \delta_{j k}\right)$ is not a complete surface.

When $a N>1$ but $a N<2$, there are still radially symmetric finite-energy $N$-string solutions. In this case the function $u$ in (114) either stays bounded or tends to $-\infty$ as $r \rightarrow \infty$. Hence $e^{\eta}$ decays at least like

$$
e^{\eta}=\mathrm{O}\left(r^{-2 a N}\right), \quad 2 a N>2
$$

as $r \rightarrow \infty$. Thus, again, the metric cannot be complete no matter how $u$ or the Higgs field $\phi$ behaves at infinity.

In summary, in the class of radially symmetric solutions, the only finite-energy solutions which are able to give rise to complete gravitational metrics are contained in those stated in Theorem 2.3.

(c) One may wonder what happens if the Einstein equations are coupled with Yang-Mills-Higgs equations with non-Abelian gauge groups. When the cosmic 
strings are sought so that the spacetime is uniform along the time axis and a vertical direction and the line element takes the specific form defined in Sect. 1, we have seen that the Einstein tensor has a significant reduction to a form that its only nonvanishing component is the Gauss curvature of a 2-surface where nontrivial geometry is coded in order to accommodate gravity. Such a property imposes a great restriction to the form of the energy-momentum tensor $T_{\mu \nu}$ via the Einstein equations

$$
G_{\mu v}-\Lambda g_{\mu v}=-4 \pi G T_{\mu v},
$$

where $\Lambda \geqq 0$ is the cosmological constant. In fact, the system (116) indicates that self-dual cosmic strings such as those contained in the Bogomol'nyi equations are to be considered to achieve consistency. This observation motivates our earlier work in [Y2]. There we found when the gauge group is $U(1)$, we must have $\Lambda=0$ which is the case studied in the present paper. When the gauge group is either $S U(2)$ or $S U(2) \times U(1)$, namely a simplified Georgi-Glashow model [AO1] or the standard Weinberg-Salam model [AO2], the cosmological constant $\Lambda$ must be positive and the associated gravitational metric can never be complete. Here we would like to remark that incompleteness of the metric is in fact a simple consequence of the positivity of $\Lambda$. Indeed, assume $\Lambda>0$. Then 00 -component of Eq. (116) reads

$$
K_{g}-\Lambda=4 \pi G \mathscr{E}
$$

which is defined an unknown 2-surface $(M, g)$ conformal to $\mathbf{R}^{2}$. Again, $K_{g}$ is the Gauss curvature of $(M, g)$. Since $\mathscr{E} \geqq 0$, Eq. (117) says that $K_{g} \geqq \Lambda>0$. By the theorem mentioned in the above Remark (a), we are convinced that $K_{g}$ is not the curvature of any complete metric of $M$. Thus, it seems to indicate that complete gravitational metrics may only arise from the Einstein equations coupled with Abelian gauge and matter fields such as those provided in Theorem 2.3.

The methods here are immediately applicable to the more general problems studied in [GORS] where the gauge and Higgs sector possesses a broken $S U(2)_{\text {global }} \times$ $U(1)_{\text {local }}$ symmetry. The accurate behavior of the metric at infinity of the solutions obtained in this paper and the solutions constructed in [CHMcY] may directly be used to determine the corresponding asymptotic deficit angles. An easy calculation shows that the solutions that approach the symmetric vacuum at infinity lead to much larger deficit angles which may occupy an entire continuum.

\section{Concluding Remarks}

In this paper, we have derived obstructions to the existence of static and regular cosmic string solutions of the coupled Einstein and Abelian Higgs equations. These obstructions are of a topological nature, which may be seen clearly when the underlying surface where the strings reside in compact, and are not present when the Einstein equations or the weak gravitational coupling is ignored. We have observed that, now, the symmetry-breaking scale, the string number, the base-space topology, and Newton's gravitational constant all play important roles. It will be interesting to know what happens in non-Bogomol'nyi coupling where $\lambda \neq 1$. We suspect that similar obstructions should occur.

The solutions constructed in this paper can indeed generate local concentrations of energy and curvature. This fact is easily seen from the properties of the solutions 
and the governing equations (1) or (5). The zeros of the Higgs field $\phi$ obviously produce energy peaks on the underlying surface $M$. Due to the first equation in (1) or (5), we observe that these energy peaks directly give rise to curvature peaks. These peaks are initial seeds for matter accretion in the theory of galaxy formation in the superconducting phase stage. Note that there are no metric singularities at all.

The paper also leaves a number of questions which will be worthwhile to pursue.

The first question is about the existence of an $N$-string solution when $N=1,2$ in a compact surface $M$ under the condition (2). Our crucial assumption (19) fails in this case. As mentioned before, when (19) is not fulfilled, the $L^{p}$-convergence with some $p>1$ of the approximation sequence constructed in the proof of Theorem 1.2 is not ensured. Thus, we encounter a gap for existence when $N=1,2$. Whether it is only a technical problem or has a hidden geometric/topological reason is not quite clear to us at this moment.

The second question concerns the obstruction (21). We have learned that (21) is necessary and sufficient for the existence of a regular static finite-energy $N$-string solution with cylindrical symmetry so that the field configurations enjoy the radial dependence as given by the standard expressions (20). It is not clear whether such a condition or any condition like that occurs for nonradial solutions. In our construction of nonradial solutions, we have seen that the stronger condition $N \leqq 1 / 2 \pi \varepsilon^{2} G$ plays an important part both in order to get local regularity (a suitable $L_{\text {loc }}^{p}$-convergence) and to get asymptotic control at space infinity in our problem. We do not know whether that condition can be lifted. Since we have obtained finite-energy radial solutions under (21), it seems reasonable to speculate that there may be nonradial solutions realizing any prescribed $N$-string distributions in the regime

$$
\frac{1}{2 \pi \varepsilon^{2} G}<N<\frac{1}{\pi \varepsilon^{2} G}
$$

as well. Several obstacles will have to be dealt with in order to move on.

The third question is whether completeness of a gravitational metric is important to cosmological consequences. Theorem 2.3 already identified a subclass of solutions which lead to complete metrics. We have seen there that, if we insist on the metric completeness, we have obtained all possible multi-string solutions for the coupled Einstein and Bogomol'nyi equations (5) so that the matter-gauge fields approach the broken vacua asymptotically.

The methods used in the present paper suggest ways to obtain multi-string solutions numerically. A similar approach has been taken for the self-dual Abelian Higgs and Chern-Simons models where solutions over the full $\mathbf{R}^{2}$ are approximated by solutions constructed on a sequence of bounded domains and the truncation errors near infinity are shown to vanish exponentially fast. It would be interesting to conduct a parallel study for computing multi-strings because in this situation we still have several unknown parameter regimes to be investigated. Here we expect that the truncation errors decay faster at infinity than any power type function of the form $r^{-b}$ for $N<1 / 2 \pi \varepsilon^{2} G$ due to Theorem 2.3. Furthermore, our discussion already provides the problem over a compact surface an implementable method to find the solutions. It may be useful to test by the iteration scheme described in Sect. 4 whether there are solutions with string charges $N=1,2$.

The study in this paper on the borderline case $N=1 / 2 \pi \varepsilon^{2} G$ also reveals an important fact that radially symmetric solutions and nonradially symmetric solutions 
may have very different features and properties. When radial symmetry is assumed so that the strings are clustered at the origin, we have seen from (85) that there is a unique solution which approaches the asymmetric vacuum at infinity so that the conformal factor $e^{\eta}$ in the gravitational metric

$$
\mathrm{d} s^{2}=-\mathrm{d} t^{2}+\mathrm{d} z^{2}+e^{\eta}\left(\mathrm{d} x_{1}^{2}+\mathrm{d} x_{2}^{2}\right)
$$

satisfies

$$
e^{\eta(x)}=2 N e^{\frac{1}{N}} r^{-2} e^{\left(u-e^{u}\right) / N}, \quad r=|x|, \quad x \in \mathbf{R}^{2},
$$

where $u=\ln |\phi|^{2}=0$ at infinity. On the other hand, for multi-string solutions ( $m \geqq 2$ ) for which the radial symmetry property is not present, the metric factor is given by

$$
e^{\eta(x)}=\lambda \prod_{l=1}^{m}\left|x-p_{l}\right|^{-2 n_{l} / N} e^{\left(u-e^{u}\right) / N}, \quad x \in \mathbf{R}^{2} .
$$

Here $\lambda>0$ should not be confused with the coupling parameter in the Abelian Higgs model. We have shown the existence of solutions for all $\lambda$ sufficiently large (see Lemma 5.2 and Subsect. 5.3). Therefore, the obstruction to an arbitrary choice of the background conformal factor (85) for the metric (118) disappears for multistrings. This fact is significant because the property $u=0$ at infinity in (119) and (120) clearly indicates that these equations give rise to different metric background at infinity. Thus we see that 'multi-strings certainly enjoy a richer spectrum of structures than radially symmetric solutions with a single string center. This interesting phenomenon does not take place in the subcritical case $N<1 / 2 \pi \varepsilon^{2} G$. In this latter case we have shown in [CHMcY] that for any $\lambda>0$ there is an $N$-string solution in the asymmetric vacuum asymptotically, i.e., $u=0$ at infinity, and the metric factor assumes the form

$$
e^{\eta}=\lambda r^{-4 \pi \varepsilon^{2} G N} e^{2 \pi \varepsilon^{2} G\left(u-e^{u}\right)}, \quad r=|x| .
$$

This result indicates the indifference of system concerning the choice of the conformal factor $\lambda^{2}$

Our prescribed string defect problem for the coupled Einstein and Abelian Higgs equations considered here may be compared with a well-developed area in differential geometry - the prescribed Gauss curvature problem on a 2-surface [A, $\mathrm{Av}, \mathrm{ChY}, \mathrm{KW} 1, \mathrm{KW} 2, \mathrm{Mc}$, Nil]. In our situation, the Gauss curvature is determined by the energy distribution of the matter and gauge interactions and the latter are strongly governed by the prescribed string defects and their associated strengths. In turn, the Gauss curvature affects the gravitational background which immediately influences the matter-gauge interactions as a feed-back.

Acknowledgements. The author would like to thank Luis Caffarelli, Poul Olesen, and Thomas Spencer for helpful conversations and comments. He would also like to thank the referee for several valuable suggestions. This work was supported in part by the National Science Foundation Grant DMS-9304580 at the Institute for Advanced Study.

\footnotetext{
${ }^{2}$ Again, in [CHMcY], the results are stated within the special scale $\lambda=1$. However, the results in the subcritical case $N<1 / 2 \pi \varepsilon^{2} G$ and supercritical case $N>1 / 2 \pi \varepsilon^{2} G$ are valid for any $\lambda>0$. This fact is crucial in our obstruction theorem in the noncompact case (Theorem 2.1) in this paper.
} 


\section{References}

[Ab] Abrikosov, A.A.: On the magnetic properties of superconductors of the second group. Sov. Phys. JETP 5, 1174-1182 (1957)

[ADN] Agmon, S., Douglis, A., Nirenberg, L.: Estimates near the boundary of solutions of elliptic partial differential equations satisfying general boundary conditions. I. Comm. Pure Appl. Math. 12, 623-727 (1959)

[AO1] Ambjorn, J., Olesen, P.: Anti-screening of large magnetic fields by vector bosons. Phys. Lett. B214, 565-569 (1988)

[AO2] Ambjorn, J., Olesen, P.: On electroweak magnetism. Nucl. Phys. B315, 606-614 (1989)

[A] Aubin, T.: Nonlinear Analysis on Manifolds: Monge-Ampére Equations. Berlin, New York: Springer, Heidelberg, 1982

[Av] Aviles, P.: Conformal complete metrics with prescribed non-negative Gaussian curvature in $\mathbf{R}^{2}$. Invent. Math. 83, 519-544 (1986)

[BMc] Bartnik, R., McKinnon, J.: Particle-like solutions of the Einstein-Yang-Mills equations. Phys. Rev. Lett. 61, 141-144 (1988)

[BJS] Bers, L., John, F., Schechter, M.: Partial Differential Equations. Providence. RI: Amer. Math. Soc., 1964

[B] Bogomol'nyi, E.B.: The stability of classical solutions. Sov. J. Nucl. Phys. 24, 449454 (1976)

[Bo] Bor, G.: Yang-Mills fields which are not self-dual. Commun. Math. Phys. 145, $393-$ 410 (1992)

[BM] Bor, G., Montgomery, R.: $S O(3)$ invariant Yang-Mills which are not self-dual. In: Hamiltonian Systems, Transformation Groups and Spectral Transform Method, University of Montréal, pp. 191-198, 1990

[Br] Bradlow, S.: Vortices in holomorphic line bundles over closed Kähler manifolds. Commun. Math. Phys. 135, 1-17 (1990)

[Bra] Brandenberger, R.H.: Cosmic strings and the large-scale structure of the universe. Phys. Scripta. T36, 114-126 (1991)

[Bu] Burzlaff, J.: Non-self dual solutions of $S U(3)$ Yang-Mills theory and a two-dimensional Abelian Higgs model. Phys. Rev. D24, 546-547 (1981)

[CY] Caffarelli, L.A., Yang, Y.: Vortex condensation in the Chern-Simons Higgs model: An existence theorem. Commun. Math. Phys., to appear

[ChY] Chang, S.Y.A., Yang, P.C.: Prescribing Gaussian curvature on $S^{2}$. Acta Math. 159, 215-259 (1987)

[CHMcY] Chen, X., Hastings, S., McLeod, J.B., Yang, Y.: A nonlinear elliptic equation arising from gauge field theory and cosmology. Proc. R. Soc. London, series A, 446, 453478 (1994)

[CG] Comtet, A., Gibbons, G.W.: Bogomol'nyi bounds for cosmic strings. Nucl. Phys. B299, $719-733$ (1988)

[Ga] Garcia-Prada, O.: A direct existence proof for the vortex equations over a compact Riemann surface. Bull. London Math. Soc. 26, 88-96 (1994)

[G] Garfinkle, D.: General relativistic strings. Phys. Rev. D32, 1323-1329 (1985)

[GOR] Gibbons, G.W., Ortiz, M.E., Ruiz, F.R.: Existence of global strings coupled to gravity. Phys. Rev. D39, 1546-1551 (1989)

[GORS] Gibbons, G.W., Ortiz, M.E., Ruiz, F.R., Samols, T.M.: Semi-local strings and monopoles. Nucl. Phys. B385, $127-144$ (1992)

[GT] Gilbarg, D., Trudinger, N.S.: Elliptic Partial Differential Equations of Second Order. Berlin, Heidelberg, New York: Springer, 1977

[Gr] Gregory, R.: Gravitational stability of local strings. Phys. Rev. Lett. 59, 740-743 (1987)

[JT] Jaffe, A., Taubes, C.H.: Vortices and Monopoles, Boston: Birkhäuser, 1980

[KW1] Kazdan, J.L., Warner, F.W.: Curvature functions for compact 2-manifolds. Ann. of Math. 99, 14-47 (1974)

[KW2] Kazdan, J.L., Warner, F.W.: Curvature functions for open 2-manifolds. Ann. of Math. 99, 203-219 (1974)

[K1] Kibble, T.W.B.: Some implications of a cosmological phase transition. Phys. Rep. 69, $183-199$ (1980) 
[K2] Kibble, T.W.B.: Cosmic strings - an overview. In: The Formation and Evolution of Cosmic Strings, ed. Gibbons, G., Hawking, and Vachaspati, T., Cambridge: Cambridge Univ. Press, 1990, pp. 3-34

[LM] Laguna-Castillo, P., Matzner, R.A.: Coupled field solutions for $U(1)$-gauge cosmic strings. Phys. Rev. D36, 3663-3673 (1987)

[L] Linet, B.: A vortex-line model for a system of cosmic strings in equilibrium. General Relat. Grav. 20, 451-456 (1988)

[Mc] McOwen, R.C.: Conformal metrics in $\mathbf{R}^{2}$ with prescribed Gaussian curvature and positive total curvature. Indiana U. Math. J. 34, 97-104 (1985)

[NS] Nash, C., Sen, S.: Topology and Geometry for Physicists. London, New York: Academic, 1983

[NO] Nielsen, H.B., Olesen, P.: Vortex-line models for dual strings. Nucl. Phys. B61, 45$61(1973)$

[Ni1] Ni, W.-M.: On the elliptic equation $\Delta u+K(x) e^{2 u}=0$ and conformal metrics with prescribed Gaussian curvatures. Invent. Math. 66, 343-352 (1982)

[Ni2] Ni, W.-M.: On the elliptic equation $\Delta u+K(x) u^{(n+2) /(n-2)}=0$, its generalizations, and applications in geometry. Indiana Univ. Math. J. 31, 493-529 (1982)

[N1] Noguchi, M.: Ph. D. Thesis, Duke University, 1985

[N2] Noguchi, M.: Yang-Mills-Higgs theory on a compact Riemann surface. J. Math. Phys. 28, 2343-2346 (1987)

[P] Parker, T.H.: Nonminimal Yang-Mills fields and dynamics. Invent. Math. 107, 397$420(1992)$

[SS] Sadun, L., Segert, J.: Non-self-dual Yang-Mills connections with quadrupole symmetry. Commun. Math. Phys. 145, 362-391 (1992)

[SSU] Sibner, L.M., Sibner, R.J., Uhlenbeck, K.: Solutions to Yang-Mills equations that are not self-dual. Proc. Natl. Acad. Sci. USA 86, 8610-8613 (1989)

[SWYMc] Smoller, J.A., Wasserman, A.G., Yau, S.-T., McLeod, J.B.: Smooth static solutions of the Einstein/Yang-Mills equations. Commun. Math. Phys. 143, 115-147 (1991)

[S] Sternberg, S.: Lectures on Differential Geometry. New Jersey: Prentice-Hall, 1964

[SY] Spruck, J., Yang, Y.: The existence of non-topological solitons in the self-dual ChernSimons theory. Commun. Math. Phys. 149, 361-376 (1992)

[T1] Taubes, C.H.: Arbitrary $N$-vortex solutions to the first order Ginzburg-Landau equations. Commun. Math. Phys. 72, 277-292 (1980)

[T2] Taubes, C.H.: On the equivalence of the first and second order equations for gauge theories. Commun. Math. Phys. 72, 207-227 (1980)

[T3] Taubes, C.H.: The existence of a non-minimal solution to the $S U(2)$ Yang-Mills-Higgs equations on $\mathbf{R}^{3}$, Parts I, II. Commun. Math. Phys. 86, 257-320 (1982)

[V] Vilenkin, A.: Cosmic strings and domain walls. Phys. Rep. 121, 263-315 (1985)

[W] Witten, E.: Superconducting strings. Nucl. Phys. B249, 557-592 (1985)

[Y1] Yang, Y.: An equivalence theorem for string solutions of the Einstein-matter-gauge equations. Lett. Math. Phys. 26, 79-90 (1992)

[Y2] Yang, Y.: Self duality of the gauge field equations and the cosmological constant. Commun. Math. Phys. 162, 481-498 (1994) 